\title{
Cooling of hypernuclear compact stars: Hartree-Fock models and high-density pairing
}

\author{
Adriana R. Raduta ${ }^{1 \star}$ Jia Jie $\mathrm{Li}^{2} \dagger$ Armen Sedrakian ${ }^{3,4} \ddagger$ and Fridolin Weber ${ }^{5,6} \oint$ \\ ${ }^{1}$ National Institute for Physics and Nuclear Engineering, RO-077125 Bucharest, Romania \\ ${ }^{2}$ Institute for Theoretical Physics, J. W. Goethe University, D-60438 Frankfurt am Main, Germany \\ ${ }^{3}$ Frankfurt Institute for Advanced Studies, D-60438 Frankfurt-Main, Germany \\ ${ }^{4}$ Institute of Theoretical Physics, University of Wroclaw, 50-2014 Wroclaw, Poland \\ ${ }^{5}$ Department of Physics, San Diego State University, 5500 Campanile Drive, San Diego, CA 92182, USA \\ ${ }^{6}$ Center for Astrophysics and Space Sciences, University of California at San Diego, La Jolla, CA 92093, USA
}

23 May 2019

\begin{abstract}
The thermal evolution of hypernuclear compact stars is studied for stellar models constructed on the basis of covariant density functional theory in Hartree and HartreeFock approximation. Parametrizations of both types are consistent with the astrophysical mass constraints on compact stars and available hypernuclear data. We discuss the differences of these density functionals and highlight the effects they have on the composition and on the cooling of hypernuclear stars. It is shown that hypernuclear stars computed with density functional models that have a low symmetry energy slope, $L$, are fairly consistent with the cooling data of observed compact stars. The class of stellar models based on larger $L$ values gives rise to the direct Urca process at low densities, which leads to significantly faster cooling. We conjecture high-density pairing for protons and $\Lambda$ 's in the $P$-wave channel and provide simple scaling arguments to obtain these gaps. As a consequence the most massive stellar models with masses $1.8 \leq M / M_{\odot} \leq 2$ experience slower cooling by hyperonic dUrca processes which involve $\Lambda$ 's and protons.
\end{abstract}

Key words: dense matter - stars: neutron - stars: thermal evolution

\section{INTRODUCTION}

The interest in the hyperonization of matter at high densities in compact stars was rekindled in the last decade by the observations of several white dwarfpulsar binaries with pulsar masses close to two solar masses (Demorest et al. 2010; Antoniadis et al. 2013; Fonseca et al. 2016; Barr et al. 2017). This problem was studied mainly within the density functional theory based on the Hartree (mean-field) approximation to obtain the equation of state (EoS) of matter (Weissenborn et al. 2012a,b; Bonanno \& Sedrakian 2012; Bednarek et al. 2012; Long et al. 2012; Colucci \& Sedrakian 2013; Miyatsu et al. 2013; Van Dalen et al. 2014; Gusakov et al. 2014; Maslov et al. 2015; Gomes et al. 2015; Oertel et al. 2015; Fortin et al. 2016; Tolos et al. 2016; Fortin et al. 2017; Marques et al. 2017; Spinella 2017; Li et al. 2018a,b; Spinella \& Weber 2018). Two solar mass neutron star

\footnotetext{
* E-mail: araduta@nipne.ro

† E-mail: jiajieli@th.physik.uni-frankfurt.de

† sedrakian@fias.uni-frankfurt.de

$\S$ fweber@sdsu.edu
}

models with a hyperonic admixture have nevertheless been constructed also within alternative frameworks, which include the quark-meson coupling model (Stone et al. 2007), the auxiliary field diffusion Monte-Carlo approach (Lonardoni et al. 2015), cluster variational method (Togashi et al. 2016; Yamamoto et al. 2017) and, more recently, Brueckner-Hartree-Fock theory (Yamamoto et al. 2017). Density functionals for hypernuclear matter and its EoS were recently constructed on the basis of Hartree-Fock theory (Li et al. 2018a,b). Including the Fock contribution in the treatment allows one to introduce the pion (and more generally tensor forces) explicitly, thus achieving a more versatile representation of the density functional. In this work we will include these EoS in our study of the cooling behavior of hypernuclear stars thus extending the pool of EoS from which our models are built (Raduta et al. 2018); hereafter Paper I. Another purpose of this work is to examine how changes in the patterns of the high-density pairing affect the cooling of hypernuclear stars. It was observed that the most massive hypernuclear stars cool very fast by the $p \Lambda$ direct Urca (hereafter dUrca) process (see Paper I). The fast cooling arises because these stars 
develop cores where protons and $\Lambda$ 's are unpaired, as their partial densities exceed the density at which the ${ }^{1} S_{0}$ pairing gap closes. As is well known, neutron matter supports ${ }^{3} P_{2^{-}}{ }^{3} F_{2}$ pairing at densities beyond the density for ${ }^{1} S_{0}$ pairing. We conjecture here that the same occurs for the protonic and $\Lambda$ components of dense matter. Below, we will use simple estimates of the gaps for protons and $\Lambda$ 's, which are based on the symmetries of nuclear and hypernuclear forces, to gauge the effect of high-density pairing on the cooling of massive hypernuclear stars.

The early studies of the cooling of hypernuclear stars did not include the high-mass astrophysical constraint on the EoS of hypernuclear matter (Haensel \& Gnedin 1994; Schaab et al. 1998; Tsuruta et al. 2009). In Paper I we started a systematic study of the cooling of hypernuclear compact stars on the basis of modern density functionals mentioned above. Several works have appeared since then in this context: Grigorian et al. (2018) used the "nuclear medium cooling" scenario (Blaschke et al. 2004; Schaab et al. 1997) to account for hyperons using an EoS based on the density functional theory (Maslov et al. 2015), pion-mediated enhanced neutrino emissivities for the modified Urca and bremsstrahlung processes, vanishing $P$-wave neutron pairing, and phenomenological crust-core temperature relation. The pion-mediated enhanced neutrino emissivities and vanishing $P$-wave neutron pairing are the key factors that distinguish their models from those in Paper I. Nevertheless, the remainder of the physical input, for example, the crust-core temperature relation, is not identical to ours. Note that Paper I does not implement the minimal cooling scenario (Page et al. 2004) as it allows for the direct dUrca process among nucleons and hyperons, uses pair-breaking processes with dressed (in-medium) vertices, and covers a wide range of masses up to the maximum mass of $M_{\max } / M_{\odot} \sim 2$, which implies a varying cooling behavior for the mass hierarchy (see, in particular, the conclusion Section of Paper I). The same problem was also addressed by Negreiros et al. (2018) using hyperonic EoS based on the FSU class of relativistic density functional models which feature massive objects and are tuned to available data on hypernuclei (Tolos et al. 2017b,a). In these models, the main cooling agents are the $(\Lambda, p)$ and $(n, p)$ dUrca processes which were regulated by variations of proton and neutron superfluid gaps. In particular the density range over which the proton $S$-wave gap is non-zero has been explored with the aim to obtain a satisfactory agreement with the data. The main difference with respect to Paper I is the absence of pairing in the hyperonic sector. Combined, the emerging new generation of cooling models of hypernuclear stars have the potential to further constraining the physics of dense nuclear matter, especially its composition.

This work is structured as follows. In Sec. 2 we review the EoS models used in our cooling simulations. We discuss the stellar parameters (mass, radius, etc.) and the range of Fermi momenta of dominant particles predicted by these models. Section 2.1 is devoted to the discussion of $P$-wave pairing of protons and $\Lambda$ 's. Simple symmetry arguments are used to extract the values and density dependences of the corresponding gaps. The critical temperatures of various species are mapped on the internal radius of the models and the pairing patterns inside the star are discussed. The results of numerical simulations of the thermal evolution of hypernuclear compact stars are shown in Section 3. A short summary of our results along with conclusions is given in Section 4.

\section{EQUATION OF STATE AND PAIRING}

We consider a set of equations of state which describe hypernuclear matter within the relativistic density functional theory (DFT) of nuclear matter. In addition to the three Hartree models that were studied in Paper I, here we add to our collection four Hartree-Fock (HF) models for the EoS of hypernuclear matter ( $\mathrm{Li}$ et al. 2018a,b). In contrast to Hartree models, the HF models account for the tensor interaction between the baryons and include $\pi$-meson exchanges in addition to the $\sigma, \omega, \rho$ and possibly $\phi$ meson exchange included in the Hartree models. The key nuclear properties of these models are displayed in Table 1 . With the nuclear saturation density $\left(n_{0}\right)$ and energy per particle $\left(E_{s}\right)$ well constrained, the list shows the range of values of the nuclear compressibility $K$, symmetry energy $J$, and its slope $L$. Note that all HF models are characterized by large values of $L$ with the PKA1 parametrization having the largest $J$ and $L$ values. These are at the boundaries inferred from experimental nuclear physics data (Tsang et al. 2012; Lattimer \& Steiner 2014). Table 2 lists the meson content of each model. The vector meson-hyperon coupling constants were chosen in each case on the basis of either the $\mathrm{SU}(3)$ or SU(6) spin-flavor symmetric quark model (see column 3 of Table 2). The coupling of the scalar meson to hyperons are tuned such as to reproduce the depths of the hyperon potential in symmetric saturated nuclear matter. DDME2, GM1A and SWL use the values $U_{\Lambda}^{(N)} \approx-28$ $\mathrm{MeV}, U_{\Xi}^{(N)} \approx-18 \mathrm{MeV}$ and $U_{\Sigma}^{(N)} \approx 30 \mathrm{MeV}$ (Gal et al. 2016), while the PK parametrizations use $U_{\Lambda}^{(N)} \approx-30$ $\mathrm{MeV}, U_{\Xi}^{(N)} \approx-14 \mathrm{MeV}$ and $U_{\Sigma}^{(N)} \approx 30 \mathrm{MeV}$. We note here the sensitivity of our results to the value of the $\Sigma$ hyperon potential in symmetric saturated nuclear matter. Changing it from $U_{\Sigma}^{(N)} \simeq 30 \mathrm{MeV}$ to the less repulsive value of $U_{\Sigma}^{(N)} \simeq 10 \mathrm{MeV}$ lowers, within DDME2, the $\Sigma^{-}$threshold below that for the $\Lambda$ hyperon (Colucci \& Sedrakian 2013; Providencia et al. 2018).

The EoS of hypernuclear matter used in the present study are displayed in the upper panel of Fig. 1. For comparison, we also show in this figure the purely nucleonic nonrelativistic microscopic EoS of Akmal et al. (1998) (APR) which is frequently used in cooling simulations of nucleonic NSs. It is seen that the EoS predicted by APR and the DDME2 and SWL models are close to each other for $n \lesssim n_{0}$, where the low- $L$ mean-field models reproduce the results of pure neutron matter (PNM) obtained by ab initio models (Fortin et al. 2016). In the density range $n_{0} \lesssim n \lesssim 4 n_{0}$ the APR EoS is softer than the relativistic DFT based EoS; at higher densities the inverse is true. The latter feature is mainly due to the fact that the relativistic DFT based EoS feature increasingly abundant hyperonic components at higher densities, which softens the EoS. A strict comparison between the models can be carried out in the case of PNM, in which case the contribution of the iso-vector sector to the EoS of different models becomes clear. At densities $n \gtrsim 2 n_{0}$ the value of the energy per neutron $(E / A)^{(\mathrm{PNM})}$ predicted 

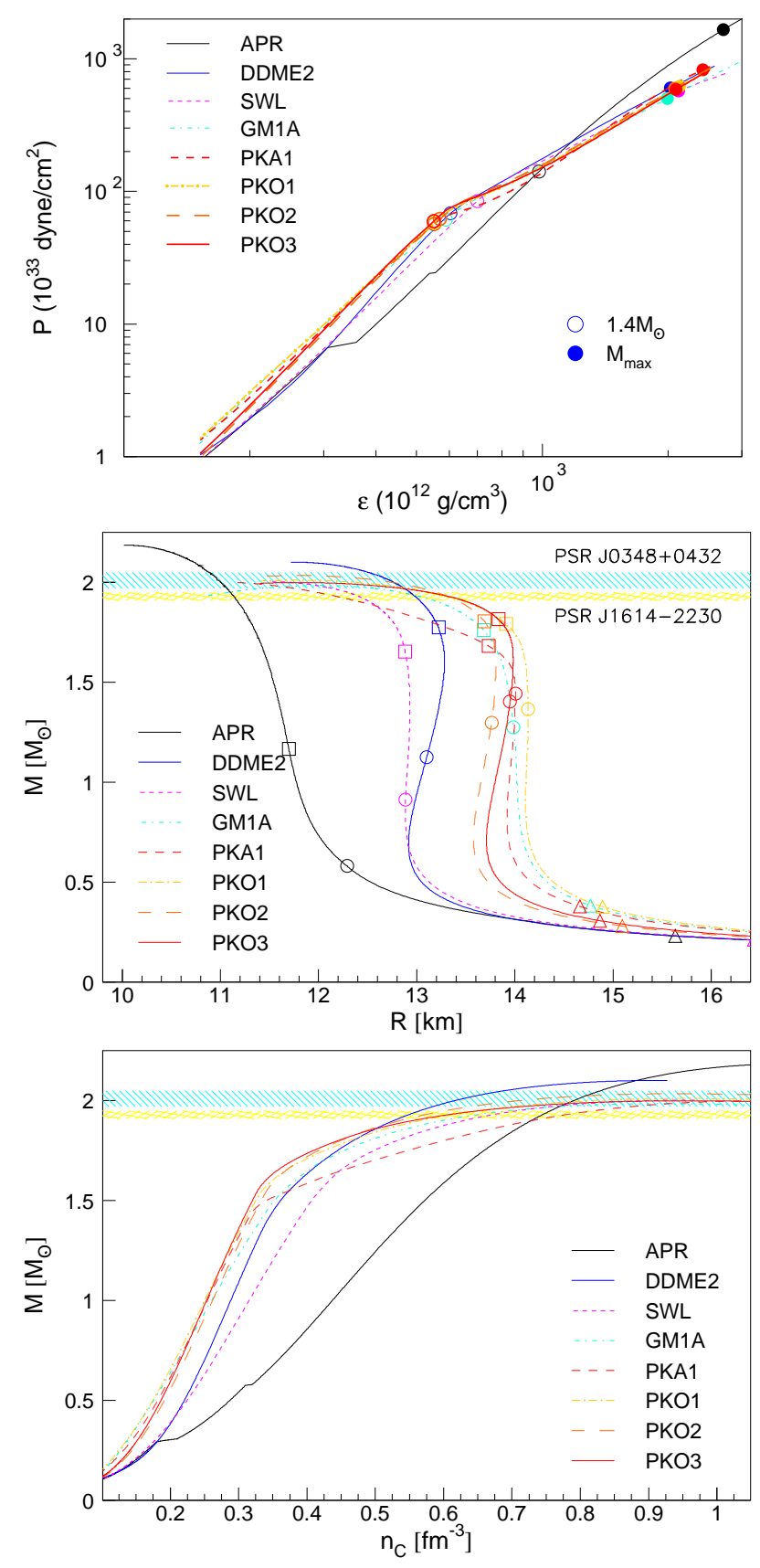

Figure 1. Equation of state (top), mass vs. radius relation (middle), and mass vs. central baryonic number density (bottom) for the stellar models considered in this work. For comparison, the predictions of the purely nucleonic APR EoS (Akmal et al. 1998) are represented as well. The solid (open) dots in the $P(\epsilon)$ plot show central values in maximum-mass $\left(1.4 M_{\odot}\right)$ neutron stars (NSs). In the $M-R$ diagram open triangles, dots and squares correspond to NS whose central densities equal $n_{0}, 2 n_{0}$, and $3 n_{0}$, respectively. The shaded horizontal stripes in the middle and bottom panels show the observed masses of pulsars PSR J0348+0432 $\left(M=2.01 \pm 0.04 M_{\odot}(\right.$ Antoniadis et al. 2013) $)$ and PSR J1614$2230\left(M=1.93 \pm 0.02 M_{\odot}(\right.$ Fonseca et al. 2016) $)$.

\begin{tabular}{lccccc}
\hline Model & $\begin{array}{c}n_{0} \\
{\left[\mathrm{fm}^{-3}\right]}\end{array}$ & $\begin{array}{c}E_{s} \\
{[\mathrm{MeV}]}\end{array}$ & $\begin{array}{c}K \\
{[\mathrm{MeV}]}\end{array}$ & $\begin{array}{c}J \\
{[\mathrm{MeV}]}\end{array}$ & $\begin{array}{c}L \\
{[\mathrm{MeV}]}\end{array}$ \\
\hline DDME2 & 0.152 & -16.1 & 250.9 & 32.3 & 51.2 \\
SWL & 0.150 & -16.0 & 260.0 & 31.0 & 55.0 \\
GM1A & 0.154 & -16.3 & 300.7 & 32.5 & 94.4 \\
PKA1 & 0.160 & -15.8 & 230.0 & 36.0 & 103.5 \\
PKO1 & 0.152 & -16.0 & 250.3 & 34.4 & 97.7 \\
PKO2 & 0.151 & -16.0 & 249.5 & 32.5 & 75.9 \\
PKO3 & 0.153 & -16.0 & 262.4 & 33.0 & 83.0 \\
APR & 0.160 & -16.0 & 266.0 & 32.6 & 57.6 \\
\hline
\end{tabular}

Table 1. Key nuclear matter properties of the relativistic DF models considered in this work. DDME2, SWL, and GM1A are relativistic Hartree models, PKA1, PKO1, PKO2, and PKO3 are Hartree-Fock models. Listed are the energy per nucleon $\left(E_{s}\right)$ and compression modulus $(K)$ at the saturation density of symmetric nuclear matter $\left(n_{0}\right)$ together with the symmetry energy $(J)$, and slope $(L)$ of the symmetry energy at $n_{0}$. The last line shows, for comparison, these quantities for the APR EoS (Akmal et al. 1998).

by the DFT models is larger than that predicted by APR. Taking as an example the DDME2 model we find that this quantity is larger by $35 \%$ at $2 n_{0}, 87 \%$ at $4 n_{0}$ and $61 \%$ at $6 n_{0}$. The EoS stiffness has important consequences for the theoretically established pressure and energy density values at the centers of compact stars. For instance, as shown in Fig. 1, for a $1.4 M_{\odot}$ star the central energy density of APR is by a factor of 1.36 to 1.73 larger than the energy densities obtained for our EoS.

The mass-radius and mass-central-density dependencies are shown in the middle and bottom panels of Fig. 1. As can be seen, all models considered in our study satisfy the $M \simeq 2 M_{\odot}$ NS mass constraint. The GM1A and PK models have $P\left(n_{B}\right)$ dependences and $L$ values close to each other so that the neutron stars with high enough $\left(n_{c} \geq 3 n_{0}\right)$ central densities have similar masses and radii. The differences just above the saturation density are reflected in variations of the radii of these models. The relativistic Hartree models DDME2 and SWL predict radii of the order of $13 \mathrm{~km}$, while the HF based models tend to predict larger radii, on the order of $14 \mathrm{~km}$. The recent detection of gravitational waves from GW170817 places an upper limit on the NS tidal deformability, and provides evidence that the radii of the merging NSs was $R \leq 13.7 \mathrm{~km}$ (Abbott \& et al. 2017; Paschalidis et al. 2018; Fattoyev et al. 2018; Annala et al. 2018; Most et al. 2018; Christian et al. 2019; Tews et al. 2019) with some uncertainty in the masses. The total mass of the binary is in the range $2.73 \leq M / M_{\odot} \leq 2.78$, which is split among the members of the binary in the range $1.36 \leq M / M_{\odot} \leq 1.6$ for one star and $1.17 \leq M / M_{\odot} \leq 1.36$ for the other. Note that the limit on the radius is extracted from the measured tidal deformability. The value of the radius is clearly related to the value of $L$, where smaller radii correspond to smaller $L$ values (see Table 2). Such correlation was observed on a much larger set of phenomenological purely nucleonic EoS by Fortin et al. (2016), who find that the correlation is stronger in less massive stars, which cover densities not much larger than $n_{0}$. Such correlation is confirmed by the fact that PK and GM1A models with their large $L$-values predict large radii. While these models overshoot the limit placed by the GW170817 analysis by about 


\begin{tabular}{|c|c|c|c|c|c|c|c|c|c|c|c|c|c|c|c|}
\hline Model & mesons & $\begin{array}{l}\text { flavor } \\
\text { sym. }\end{array}$ & $\begin{array}{c}n_{\max } \\
{\left[\mathrm{fm}^{-3}\right]}\end{array}$ & $\begin{array}{c}M_{\max }^{Y} \\
{\left[M_{\odot}\right]}\end{array}$ & $Y_{1}$ & $\begin{array}{c}n_{Y_{1}} \\
{\left[\mathrm{fm}^{-3}\right]}\end{array}$ & $\begin{array}{l}M_{Y_{1}} \\
{\left[M_{\odot}\right]}\end{array}$ & $Y_{2}$ & $\begin{array}{c}n_{Y_{2}} \\
{\left[\mathrm{fm}^{-3}\right]}\end{array}$ & $\begin{array}{l}M_{Y_{2}} \\
{\left[M_{\odot}\right]}\end{array}$ & $Y_{3}$ & $\begin{array}{c}n_{Y_{3}} \\
{\left[\mathrm{fm}^{-3}\right]}\end{array}$ & $\begin{array}{l}M_{Y_{3}} \\
{\left[M_{\odot}\right]}\end{array}$ & $\begin{array}{c}n_{\mathrm{DU}} \\
{\left[\mathrm{fm}^{-3}\right]}\end{array}$ & $\begin{array}{l}M_{\mathrm{DU}} \\
{\left[M_{\odot}\right]}\end{array}$ \\
\hline DDME2 & $\sigma, \omega, \phi, \rho$ & $\mathrm{SU}(6)$ & 0.93 & 2.12 & $\Lambda$ & 0.34 & 1.39 & $\Xi^{-}$ & 0.37 & 1.54 & $\Sigma^{-}$ & 0.39 & 1.60 & - & - \\
\hline GM1A & $\sigma, \omega, \phi, \rho$ & $\mathrm{SU}(6)$ & 0.92 & 1.99 & $\Lambda$ & 0.35 & 1.49 & $\Xi^{-}$ & 0.41 & 1.67 & - & - & - & 0.28 & 1.10 \\
\hline PKA1 & $\sigma, \sigma^{*}, \omega, \phi, \rho, \pi$ & $\mathrm{SU}(3)$ & 1.09 & 2.01 & $\Lambda$ & 0.32 & 1.43 & $\Sigma^{-}$ & 0.39 & 1.57 & $\Xi^{-}$ & 0.43 & 1.63 & 0.25 & 0.98 \\
\hline PKO1 & $\sigma, \sigma^{*}, \omega, \phi, \rho, \pi$ & $\mathrm{SU}(3)$ & 0.96 & 2.02 & $\Lambda$ & 0.33 & 1.52 & $\Xi^{-}$ & 0.58 & 1.87 & - & - & - & 0.25 & 1.01 \\
\hline PKO3 & $\sigma, \sigma^{*}, \omega, \phi, \rho, \pi$ & $\mathrm{SU}(3)$ & 0.97 & 2.02 & $\Lambda$ & 0.33 & 1.55 & $\Sigma^{-}$ & 0.45 & 1.81 & $\Xi^{-}$ & 0.48 & 1.84 & 0.28 & 1.22 \\
\hline
\end{tabular}

Table 2. Astrophysical characteristics of the relativistic DF EoS models (with hyperons) used in this work: $n_{\max }$ shows the central densities of the maximum-mass $\left(M_{\max }^{Y}\right)$ hyperonic star of each stellar sequence, $n_{Y_{i}}$ shows the threshold densities at which hyperons of type $Y_{i}$ are produced, and $M_{Y_{i}}$ lists the mass of the hyperonic star for that central density. The last two entries show the baryon number density $\left(n_{D U}\right)$ beyond which the nucleonic dUrca process is allowed in purely nucleonic NS matter and the mass $\left(M_{D U}\right)$ of the associated compact star.

$10 \%$, this discrepancy is not significant in the context of cooling. Indeed, the radius enters explicitly only the photon luminosity from the surface of the star which is unimportant for our discussion; the variations in the internal composition and distribution of particles over the internal radius are small, leading to $10 \%$ change in the radius value. The bottom panel of Fig. 1 shows that those EoS which are soft up to densities $\lesssim 4 n_{0}$ produce intermediate mass NS with high-density cores.

A more quantitative overview of the properties of hypernuclear neutron stars is given in Table 2 for the EOS of this work. The central density of the maximum mass star (fourth column), the value of the maximum mass (fifth column) and the density at which the first hyperon species appears (columns 6 and 7) are remarkably close to each other (exceptions are $n_{\max }$ for PKA1 and $n_{Y 1}$ for the SWL model). The differences in the models are larger for the remaining entries of the table, as these are controlled by the high-density behavior of the EoS. An important difference concerns the onset of the nucleonic dUrca process (column 15 of Table 2). DDME2 does not allow for this process in stable stars. The SWL models only allow for it at very high densities $(n \geq 0.9$ $\mathrm{fm}^{-3}$ ). Finally the other models predict considerably lower dUrca thresholds $\left(\sim 0.3 \mathrm{fm}^{-3}\right)$. We also note the (negative) correlation between the value of $L$ and the threshold density of the Urca process (column 15 of Table 2). For purely nucleonic models, such correlation was investigated by Fortin et al. (2016) on a larger set of density functional models which produce $2 M_{\odot}$ NS. These authors find that: (i) for $L \geq 60 \mathrm{MeV}$, there is a clear anti-correlation between $L$ and $n_{D U}$, and (ii) for $L<60 \mathrm{MeV}$ the situation is ambigous as some models allow the dUrca to operate in stars as small as $1.5 M_{\odot}$ while others forbid it in all stable configurations. Clearly, other factors play in this situation a decisive role.

Furthermore, it is important to discuss the particle content of the models under consideration, as the thermal evolution significantly depends on the composition of matter. Rather than showing the abundances of particles [see, for instance, Paper I and Li et al. (2018a,b)], we show in Fig. 2 the Fermi momenta of the most abundant particle species, neutrons (top), protons (middle) and $\Lambda$ (bottom). We also show the range of momenta over which neutron $P$-wave pairing (Ding et al. 2016), and proton $S$-wave pairing (Chen et al. 1993) and $\Lambda S$-wave pairing (Raduta et al. 2018) occur. Thus, the figure shows in a transparent manner the existence of various condensates in the centers of NSs. The suppression of pairing in any one of these channels leads to significantly faster stellar cooling.

It has been known for a long time that the cooling simulations are sensitive to the choice of the gaps of fermionic constituents of NS matter, for an overview see (Sedrakian \& Clark 2018). We now comment briefly on the gap choices made in this work. For the neutron ${ }^{3} P_{2^{-}}{ }^{3} F_{2^{-}}$ wave gap we have implemented the results of Ding et al. (2016), specifically those that have been obtained using the Argonne v18 (Wiringa et al. 1995) interaction. This calculation includes both the short- and long-range correlations in neutron matter as well as three-body contributions. Because the ${ }^{3} P_{2}{ }^{3} F_{2}$ pairing occurs at high densities the bare interaction is not constrained by the phase-shift analysis and the results corresponding to different interactions may differ in the high-density segment relevant for neutron stars. In addition, the three-body force produces a non-negligible effect. The many-body scheme used by Ding et al. (2016) is one of the most reliable to date. We will also use alternatively the result obtained within the BCS scheme with bare twobody interaction in combination with a phase-shift equivalent interaction (Baldo et al. 1998). For the proton $S$-wave gaps we use the results of Chen et al. (1993) which are based on the matrix elements extracted from Reid-soft-core potential (Chao et al. 1972). Our choice, in this case, is motivated by the fact that the corresponding gap, in its magnitude and density range, is the largest and broadest in the literature, i.e., it maximizes the effect of proton pairing on the physics studied. In particular, this choice very efficiently slows down the cooling induced by $(\Lambda, p)$ dUrca process [see Paper I for extensive discussion]. Finally, $\Lambda S$-wave pairing gaps where obtained by solving the BCS equation for hyperonic pairing, as in Paper I. For more details on $\Lambda$-pairing, see the comments on Fig. 3.

As shown in the top panel of Fig. 2, the neutron Fermi momenta in the centers of NSs computed for the low- $L$ EoS of our collection, i.e., DDME2 and SWL, are larger than the Fermi momenta obtained for the large- $L$ EoS. This follows from the fact that the former EoS produce stars that are more compact (for the same mass). For example, we find that for a $M / M_{\odot}=1.0$ mass star the central neutron densities obtained for the low- $L$ EoS are $n_{c, n}^{\text {DDME2 }}=0.26$ $\mathrm{fm}^{-3}$ and $n_{c, n}^{\mathrm{SWL}}=0.29 \mathrm{fm}^{-3}$, whereas for the large- $L$ EoS $n_{c, n}^{\mathrm{PK}} \simeq 0.22 \mathrm{fm}^{-3}$. The same values for a $M / M_{\odot}=1.8$ 


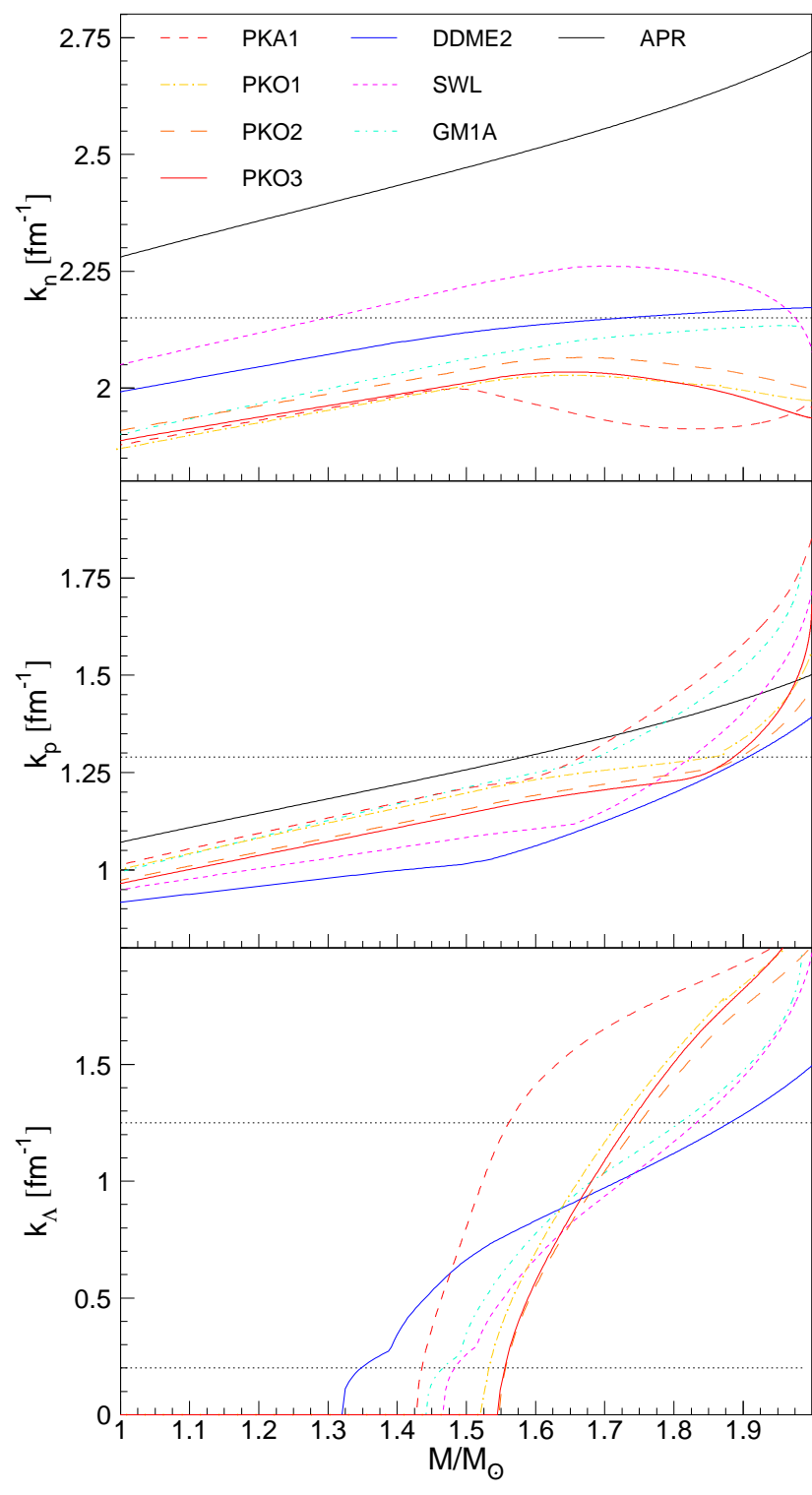

Figure 2. Fermi momenta of neutrons (top), protons (middle) and $\Lambda$ (bottom) in the centers of NS of different masses, for the collection of EoS of this work. The horizontal dotted lines mark the upper limits (top and middle panel) or the boundaries (bottom panel) of the momentum range for which neutron, proton and $\Lambda$ pairing occurs. Neutron $P$-wave pairing results are from Ding et al. (2016). For proton $S$-wave pairing we used results from Chen et al. (1993). The $\Lambda S$-wave pairing is obtained from the BCS equation (see text for details).

mass star are $n_{c, n}^{\mathrm{DDME} 2}=0.33 \mathrm{fm}^{-3}, n_{c, n}^{\mathrm{SWL}}=0.38 \mathrm{fm}^{-3}$ and $0.23 \leq n_{c, n}^{\mathrm{PK}} \leq 0.29 \mathrm{fm}^{-3}$. Note that the neutron abundance does not increase necessarily with the mass of the star (which is the case for DDME2 and GM1A), as the particle abundances are regulated in a convoluted manner by the conditions of $\beta$-equilibrium and charge neutrality. The range of momenta below which neutron ${ }^{3} P_{2^{-}}{ }^{3} F_{2}$ superfluidity exists according to Ding et al. (2016) when neutron-neutron interaction is described by the Argonne v18 (Wiringa et al. 1995) is marked by a dotted line (the lower limit is at 1.15 $\mathrm{fm}^{-1}$ and is outside the figure's scale). It is seen that neu- tron ${ }^{3} P_{2}{ }^{3} F_{2}$-wave superfluidity always extends to the center of a star of given mass for all large- $L$ EoS, whereas massive stars based on DDME2 $\left(M / M_{\odot} \geq 1.7\right)$ and SWL $\left(1.3 \leq M / M_{\odot} \leq 1.95\right)$ may feature normal neutron fluids in their centers. We also note that the large difference between the DFT based EOSs and the APR EOS is inherent in these models and not attributable to the fact that the APR EoS does not include high-density hyperonic components.

The middle panel of Fig. 2 shows the proton Fermi momentum at the star's center for our collection of EoS, which increases with the mass of the NS. The variations in the proton fraction reflects the uncertainty in the behavior of the symmetry energy away from the saturation density. Not surprizingly, a correlation is observed between the $L$ value and the proton fraction in the cores of NSs, such that the EOS with the lowest $L$ value leads to the lowest proton abundance in the core. It is also remarkable that all models show a change in the slope, which reflects a rapid increase of proton fraction as the mass increases. The upper limit on the momenta where proton $S$-wave superfluidity exists according to Chen et al. (1993) is shown by the horizontal line. Clearly, the low-mass stars feature proton ${ }^{1} S_{0}$ superfluidity which extends all the way to the stellar center, whereas in more massive stars, with $M / M_{\odot} \geq 1.6$, the $S$-wave proton pairing vanishes at some density which is below the central density. This is a crucial feature that accelerates the cooling of massive stars. We will return to this problem below.

The bottom panel in Fig. 2 shows the Fermi momentum of $\Lambda$ hyperons. The DDME2 and SWL EoS predict low $\Lambda$ fractions in massive stars, but there is no clear distinction between the EoS at intermediate stellar masses. The momentum range for which $\Lambda S$-wave superfluidity exists is bracketed by horizontal lines. Thus, we observe that the intermediate mass stars contain a $\Lambda S$-wave superfluid over the whole volume, whereas the most massive stars have cores containing either normal $\Lambda$ 's or $\Lambda$ 's paired through a higher partial wave, as discussed below.

Figure 3 shows $S$-wave pairing gaps for neutrons, protons, and $\Lambda$ 's, which have been used in our cooling simulations. The two models used for neutron $S$-wave pairing reflect the uncertainties in the $S$-wave gap related to treatment of correlations among neutrons. The maximum value of the $S$-wave gap when both short- and long-range correlations are taken into account (Ding et al. 2016) is more than twice as large as the maximum value obtained when only long-range correlations (polarization effects) are included (Schwenk et al. 2003). Because the neutron-neutron interaction at low energies is constrained by the scattering data the main uncertainty in the value of the gap arises from the many-body treatment and not from the bare interaction. See Sedrakian \& Clark (2018) for a recent discussion. The main effect of neutron $S$-wave pairing in the crust is to regulate the thermalization of the crust. In the absence of the nucleonic dUrca process, a larger gap in the crust enhances the cooling rate. The opposite effect occurs if the nucleonic dUrca operates. Neutron pairing in the crust impacts the cooling mainly over a time span of $t \lesssim 10^{2}$ yr, during which the star is not yet isothermal and the surface temperature is controlled by the crust. Therefore, the variations in the gap value are not significant for the interpretation of the data. The proton $S$-wave pairing gap, which is non-zero in the star's core, plays an important role for regulating dUrca 


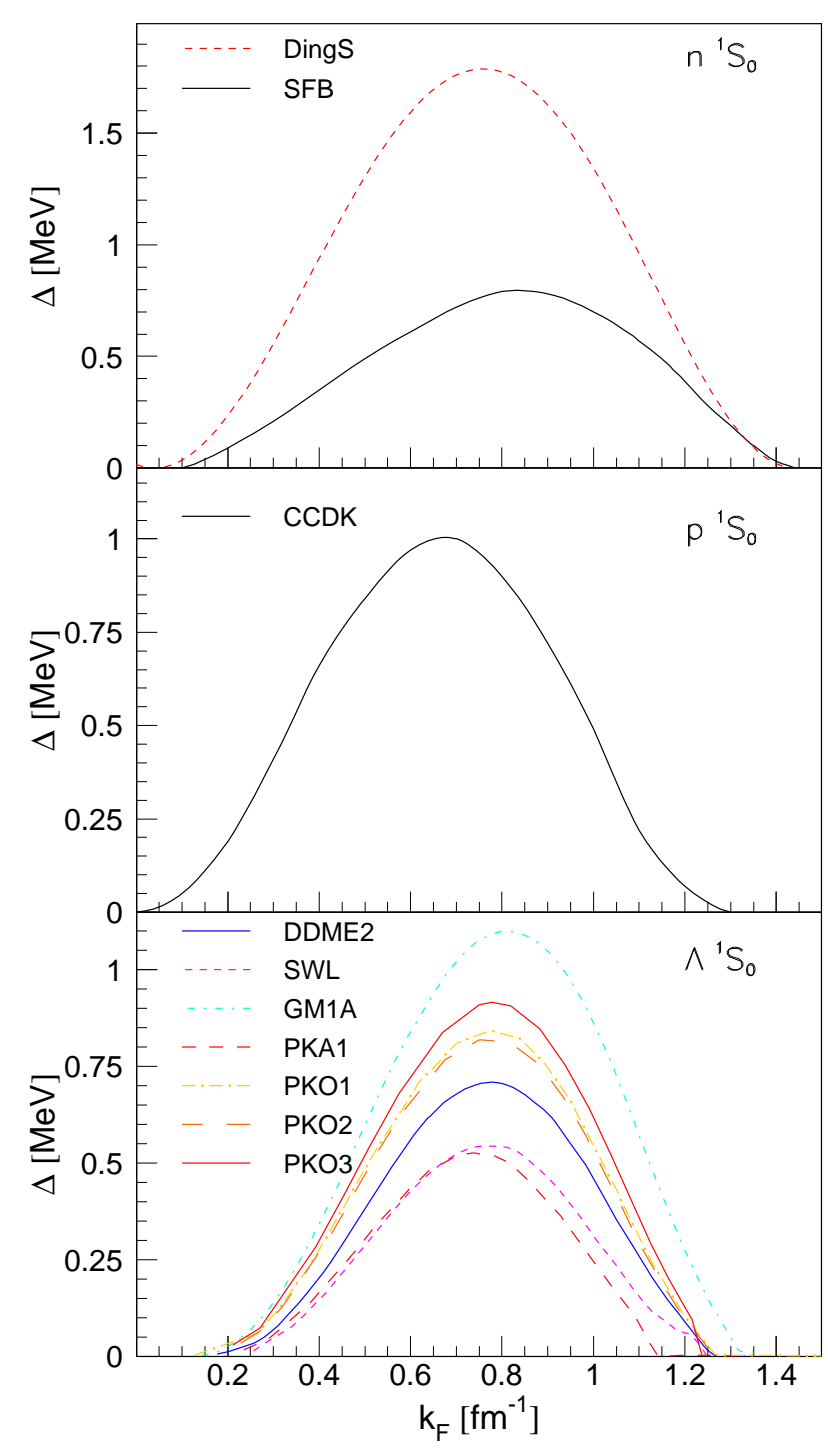

Figure 3. $S$-wave pairing gaps for neutrons (top), protons (middle) and $\Lambda$ 's (bottom) as a function of Fermi momentum. The SFB neutrons gaps (Schwenk et al. 2003) include long-range correlations only, whereas long- and short-range correlations are included in the DingS gap (Ding et al. 2016). For protons, we show the ${ }^{1} S_{0}$ gap as computed by Chen et al. (1993). The $\Lambda{ }^{1} S_{0}$ gaps are obtained as in Paper I with the bare $\Lambda-\Lambda$ interaction taken to be the configuration space parametrization of the ESC00 potential (Rijken 2001) given by Filikhin \& Gal (2002). The composition of matter itself and the $\Lambda$ single-particle spectra are computed for the EoS listed in the bottom panel.

processes involving protons. In particular, this pairing gap regulates the $\Lambda p$ channel of the hyperonic dUrca process, as discussed in Paper I and by Grigorian et al. (2018) and Negreiros et al. (2018). Here we adopt the proton gap of Chen et al. (1993) which covers the largest density range and has a relatively large maximum of the order of $1 \mathrm{MeV}$. Thus, the suppression effects through the proton $S$-wave gap will be maximized in our cooling simulations.

We have (re)computed the $\Lambda{ }^{1} S_{0}$ pairing gaps within the standard BCS theory, as described in Paper I. As in Paper I, for the bare $\Lambda-\Lambda$ interaction we used the configuration

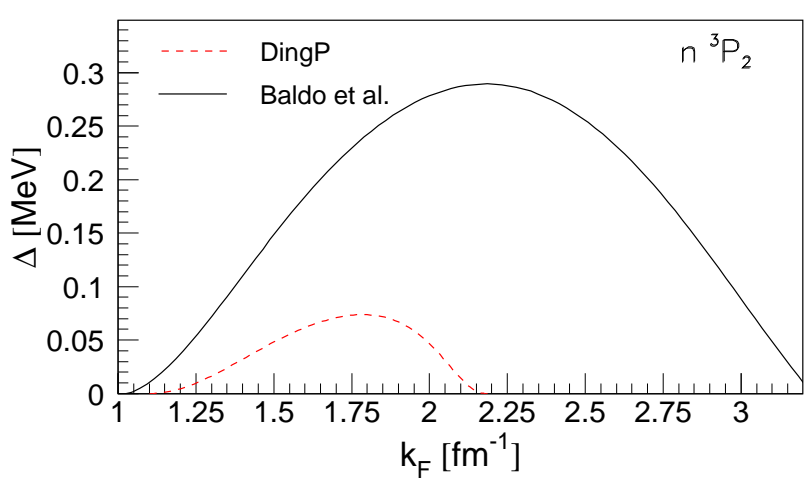

Figure 4. Neutron ${ }^{3} P_{2^{-}}{ }^{3} F_{2}$ pairing gaps as a function of Fermi momentum. The results of Ding et al. (2016) which includes short- and long-range correlations is shown by the dashed line, the result of Baldo et al. (1998) which is based on BCS theory is shown by the solid line.

space parametrization of the ESC00 (Rijken 2001) provided by Filikhin \& Gal (2002). In computing the gaps we use the background matter composition and single particle spectrum of $\Lambda$ 's in the normal state provided by the EoS under consideration. The EoS-dependence of the gap enters via the Dirac effective mass. The reduction of the hyperon masses by medium effects diminishes the density of states and, thus, the gap at the Fermi surface. ESC00 (Rijken 2001) gives for $\Lambda-\Lambda$ one of the most attractive interactions in the literature. The suppression of dUrca processes involving $\Lambda$ 's is thus maximized. Note that the $\Lambda S$-wave superfluidity occurs for Fermi momenta $0.2 \leq k_{F_{\Lambda}} \leq 1.3 \mathrm{fm}^{-1}$, which is about the same range as for $S$-wave neutron and proton condensates (see Fig. 3). It is also seen that the gaps are in the range $0.5 \leq \Delta_{\Lambda} \leq 1.2 \mathrm{MeV}$, which is comparable to the proton pairing gap. We thus anticipate a competition of these two pairing channels when considering the suppression of the $\Lambda p$ channel of the hyperonic Urca process.

The $P$-wave pairing of neutrons is harder to compute as the interactions are not constrained by the phase-shift analysis in the high-energy domain needed for this channel. To illustrate the range of theoretical possibilities we present two models of the ${ }^{3} \mathrm{P}_{2}{ }^{3} \mathrm{~F}_{2}$ pairing gap, which are shown in Fig. 4. The first choice corresponds to computations of Ding et al. (2016), already discussed in connection with Fig. 2. They use the Argonne v18 (Wiringa et al. 1995) interaction and account for short- and long-range correlations. The gap covers the Fermi-momentum range $1.15 \leq k_{F} \leq 2.1$ $\mathrm{fm}^{-1}$. Its maximum, $\Delta \simeq 0.074 \mathrm{MeV}$, occurs for $k_{F}=1.8$ $\mathrm{fm}^{-1}$. This calculation is taken as a lower bound on the gap. As an upper limit on the ${ }^{3} P_{2}{ }^{3} F_{2}$-wave pairing gap we adopt the result of Baldo et al. (1998), which corresponds to the CD Bonn (Machleidt et al. 1996) interaction and the Bruckner-Hartree-Fock spectrum. The gap covers a significantly broader range of momenta. The maximum of this gap $\left(\sim 0.3 \mathrm{MeV}\right.$ at $\left.k_{F} \sim 2.15 \mathrm{fm}^{-1}\right)$ is a factor of four larger than the gap of Ding et al. (2016). In passing we note that a number of studies of the cooling of NSs assumed a vanishing ${ }^{3} P_{2^{-}}$ ${ }^{3} F_{2}$ pairing gap [see Beznogov et al. (2018); Grigorian et al. (2018); Negreiros et al. (2018)]. This assumption improves the agreement of the cooling curves with the data on surface temperatures of some compact objects, but it may lack any 
theoretical underpinning. In one of the scenarios considered in this paper, we shall make this assumption as well.

\subsection{BCS $P$-wave pairing for protons and $\Lambda$ 's}

We have previously addressed the Bardeen-CooperSchrieffer (BCS) pairing among hyperons in the low-density limit, where the most attractive partial wave is the ${ }^{1} S_{0}$ channel. In this case hyperons are paired in spin-singlet Cooper pairs. Note that within the hypernuclear mixture of particle, pairing among the different species is strongly disfavored by the particle mass differences (except for neutrons and protons, in which case the pairing is suppressed by their large difference in number density).

The ${ }^{1} S_{0}$ gaps for $\Lambda$ hyperons were computed using the non-relativistic BCS equation for a given hyperonhyperon potential, whereas the single-particle energies and particle composition were computed for the relativistic DF method, as described in Paper I. [See also the related earlier work on hyperonic pairing by Balberg \& Barnea (1998), Wang \& Shen (2010) and Takatsuka et al. (2001).] Computing the single-particle energies and pairing gaps for different theories is not consistent formally, but in practice such an approach has been proven to work well for finite nuclei (Vretenar et al. 2005; Kucharek \& Ring 1991). It is also in line with the so-called decoupling approximation which has been applied to the many-body treatment of nuclear pairing [for a review, see (Sedrakian \& Clark 2018)]. We recall that for the $\Lambda \Lambda$ pairing interaction we used the configuration-space parameterization of the ESC00 potentials (Rijken 2001) given by Filikhin \& Gal (2002). The potential was chosen in such a manner as to maximize the pairing gap, i.e., to provide an upper limit on the values of the gap function. Note also that the $\Sigma \Sigma$ pairing was disregarded, though this possibility has been previously considered (Vidaña \& Tolós 2004) and gaps as large as several $\mathrm{MeV}$ have been obtained.

On the basis of the (approximate) isospin invariance of nuclear forces, it is natural to anticipate that ${ }^{3} P_{2-}{ }^{3} F_{2}$ pairing should also occur in the proton component, for the range of densities where this channel dominates the attraction among protons. To get an estimate of pairing gap of this channel, we account for the difference between the density of states of neutrons and protons, which amounts to replacing the neutron Landau mass $m_{n}^{*}$ by the proton Landau mass $m_{p}^{*}$. This implies a rescaling of the dimensionless pairing interaction by the factor $\alpha_{p}^{-1}=m_{p}^{*} / m_{n}^{*}$. The result is the weak-coupling estimate for the $P$-wave pairing gap for protons as

$$
\Delta_{p}=\epsilon_{F_{p}}\left(\frac{\Delta_{n}}{\epsilon_{F_{n}}}\right)^{\alpha_{p}}
$$

where $\epsilon_{F_{i}}$ is the Fermi energy of baryon $i$. Thus, from the $P$-wave pairing gap for neutrons and the Fermi energies of particles, we obtain an estimate of the $P$-wave pairing gap for protons. Equation (1) follows directly from the BCS formula $\Delta_{i}=\epsilon_{F i} \exp \left[-1 /\left(\nu_{i} V_{i}\right)\right]$, where $\nu_{i}$ and $V_{i}$ are the density of states and the pairing matrix element for a baryon of type $i$.

We further speculate about $P$-wave pairing among $\Lambda$ 's. An estimate of this pairing gap follows from the strategy adopted for protons, except that now we additionally rescale the pairing interaction among neutrons by a factor of $2 / 3$

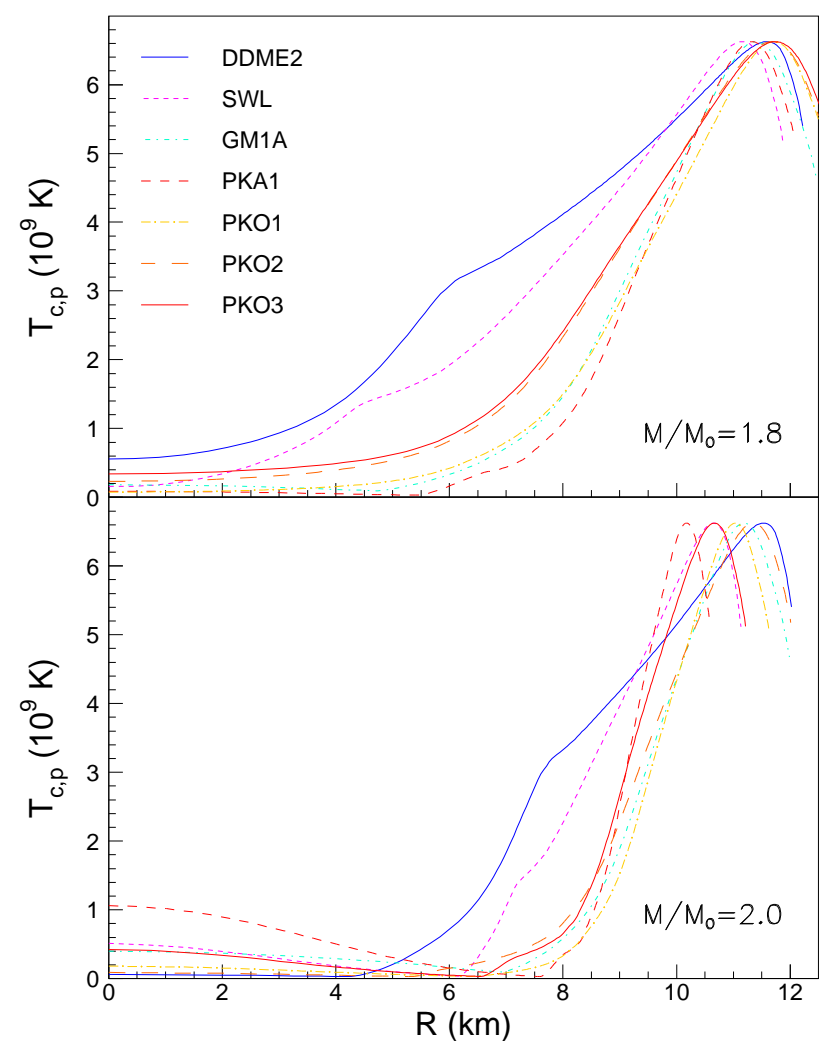

Figure 5. Critical temperature of proton superfluidity for NSs with masses of $1.8 M_{\odot}$ (top) and $2 M_{\odot}$ (bottom), for the EoS of this work. ${ }^{1} S_{0}$ pairing is modeled as in the paper of CCDK (Chen et al. 1993). The proton $P$-wave $T_{c}$ is obtained by rescaling the neutron $P$-wave gap, given in Baldo et al. (1998), via Eq. (1).

according to the SU(6) flavor quark model. This means that the dimensionless pairing interaction is rescaled by the factor $\alpha_{\Lambda}^{-1}=2 m_{\Lambda}^{*} / 3 m_{n}^{*}$ and, therefore,

$$
\Delta_{\Lambda}=\epsilon_{F_{\Lambda}}\left(\frac{\Delta_{n}}{\epsilon_{F_{n}}}\right)^{\alpha_{\Lambda}}
$$

Figures 5 and 6 show the critical temperature profiles for protons and $\Lambda$ 's inside of NSs with masses of $1.8 M_{\odot}$ (top) and $2 M_{\odot}$ (bottom) built using different EoS. For the proton ${ }^{1} S_{0}$-pairing we adopt the results of Chen et al. (1993). The $\Lambda{ }^{1} S_{0}$-pairing is determined, for each EoS, by solving the BCS equation, as explained before. The ${ }^{3} P_{2^{-}}{ }^{3} F_{2}$-pairing gap of protons and $\Lambda$ 's are obtained by the re-scaling procedure described just above, using Eqs. (1) and (2). In both cases, the results of Baldo et al. (1998) are used for the neutron ${ }^{3} \mathrm{P}_{2^{-}}{ }^{3} \mathrm{~F}_{2}$-pairing. High density pairing gaps based on the ${ }^{3} P_{2}-{ }^{3} F_{2}$ gap by Ding et al. (2016) (not illustrated) feature similar or lower critical temperatures in the innermost core, depending on EoS and NS mass. The double-bell shaped structures arise, in the case of $2 M_{\odot} \mathrm{NS}$ and, for some EoS, also $1.8 M_{\odot}$ NS, because of the two ( $S$ - and $P$-wave) pairing channels, each channel giving rise to a maximum. The maximum appearing for large internal radius corresponds to the $S$-wave pairing, whereas the one appearing for small internal radius - to the $P$-wave pairing. Very often and especially in the most massive stars, there is a shell, between those 


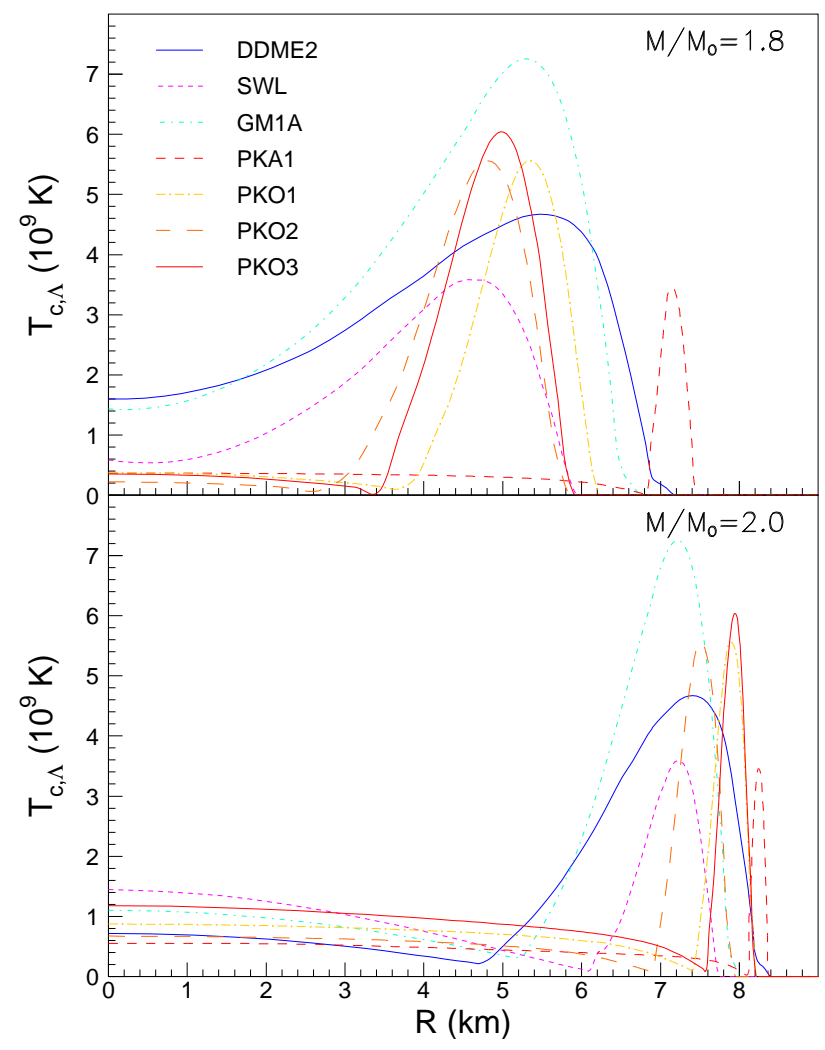

Figure 6. Critical temperature of $\Lambda$ superfluidity for stars with masses of $1.8 M_{\odot}$ (top) and $2 M_{\odot}$ (bottom), for the EoS of this work. The ${ }^{1} S_{0}$ pairing is treated in the BCS theory, as in Paper I. The critical temperature of the $P$-wave superfluid is obtained by re-scaling the neutron $P$-wave gap (Baldo et al. 1998) via Eq. (2).

featuring $S$ - and $P$-wave condensates, where pairing almost vanishes. It is straightforward to anticipate that its role in cooling will be significant.

Because of their low density, one could expect that the protons manifest ${ }^{1} S_{0}$ superfluidity in the entire stellar core. Fig. 5 shows that this is not true. For massive stars and most EoS protons are superfluid only in the outer core. When they are additionally allowed to pair in the ${ }^{3} P_{2}{ }^{3} F_{2}$ channel, the situation does not improve much as, very often, in the inner most regions the critical temperature is so low that it falls below the stellar temperature. In this event proton superfluidity will not be experienced and it will play no role in the suppression of neutrino radiation from the stellar core. Thus, while $P$-wave pairing of protons could in principle provide an efficient mechanism for the suppression of neutrino radiation, its actual occurrence depends on the adopted EoS and on the density range covered by the mass model. Note that the critical temperature for the $S$-wave pairing has a maximum value of $T_{c p} \simeq 7 \times 10^{9} \mathrm{~K}$, whereas for $P$-wave pairing this is of the order of $10^{9} \mathrm{~K}$ or less. We conclude that for most of the EoS of this study there exist domains, either in the inner core or at the boundary between inner and outer core, where $T_{c, p}$ is too low to efficiently suppress cooling via dUrca process involving protons.

For both NS masses considered in Fig. $6 \Lambda$ hyperons are in the $S$-wave superfluid state in the outer core.
For PKA1, which predicts the highest $\Lambda$ densities, the superfluid shell is very narrow. $T_{c, \Lambda}$ ranges in the interval $3-7 \times 10^{9} \mathrm{~K}$ and strongly depends on the EoS, the star's mass and the distance from the center. For DDME2 and GM1A $S$-superfluidity is encountered also in the inner core of the $1.8 M_{\odot}$ star. $\Lambda P$-wave pairing leads to critical temperatures higher than those obtained for protons. The radial changes of $T_{c, \Lambda}$ can be attributed to the different fractions and effective masses of the $\Lambda$ particle, as predicted by different EoS. For a large enough $\Lambda$ population we find a $P$-wave $\Lambda$ superfluid in the inner core for a number of models. The values of $T_{c, \Lambda}$ are typically between $2 \times 10^{8}$ and $1.5 \times 10^{9} \mathrm{~K}$.

In conclusion, it is clearly seen that in the absence of proton and $\Lambda P$-wave pairing, NSs cool rapidly (as already demonstrated in Paper I) via the dUrca process $\Lambda \rightarrow p+$ $l+\bar{\nu}_{l}$, where $l$ stands for a lepton. This will be different if high-density $P$-wave pairing of protons and $\Lambda$ 's occurs, which suppresses the dUrca process (see Sec. 3 below for explicit examples).

\section{THERMAL EVOLUTION OF HYPERNUCLEAR STARS}

We have performed a large number of cooling simulations of stellar models based on our EoS collection of hypernuclear stars described above. We assume spherically symmetric configurations of isolated self-gravitating NSs which are non-rotating and non-magnetized. Heating sources were not considered. Our collection of EoS was supplemented with the crust EoS of Negele \& Vautherin (1973) and Haensel et al. (1989), which is smoothly joined together with the core EoS. The outer envelope of the star is assumed to consist of Fe. Note that young stars are believed to have $\mathrm{He}$ or $\mathrm{C}$ atmospheres. Light-element envelopes lead to surface temperatures which are higher than those produced by heavyelement envelopes. This means that the use of a Fe envelope throughout the entire evolution artificially diminishes the effective temperature of young stars and partially compromises the agreement with the X-ray data on their thermal emission.

Our models were evolved in time from an arbitrary radial temperature profile under the assumption that the structure of the models does not change in time. This initial distribution is such that the temperature smoothly decreases from $10^{10} \mathrm{~K}$ in the center to $8 \cdot 10^{9} \mathrm{~K}$ at the boundary between the inner and outer crust and, finally, to $5 \cdot 10^{9} \mathrm{~K}$ at the surface.

As in Paper I, we employ an extension of the public domain NSCool code ${ }^{1}$ to include the hyperonic component with its radiation processes. These include the hyperonic

1 www.astroscu.unam.mx/neutrones/NSCool 
dUrca processes (Prakash et al. 1992)

$$
\begin{aligned}
\Lambda & \rightarrow p+l+\bar{\nu}_{l}, \\
\Sigma^{-} & \rightarrow\left(\begin{array}{c}
n \\
\Lambda \\
\Sigma^{0}
\end{array}\right)+l+\bar{\nu}_{l}, \\
\Xi^{-} & \rightarrow\left(\begin{array}{c}
\Lambda \\
\Xi^{0} \\
\Sigma^{0}
\end{array}\right)+l+\bar{\nu}_{l}, \\
\Xi^{0} & \rightarrow \Sigma^{+}+l+\bar{\nu}_{l},
\end{aligned}
$$

where $l$ stands for a lepton, either electron or muon, and $\bar{\nu}_{l}$ is the associated anti-neutrino. In addition, we include the neutrino emission via the Cooper pair-breaking and formation $(\mathrm{PBF})$ mechanism:

$$
\{Y Y\} \rightarrow Y+Y+\nu+\bar{\nu}, \quad Y+Y \rightarrow\{Y Y\}+\nu+\bar{\nu},
$$

where $\{Y Y\}$ stand for a hyperonic Cooper pair. The PBF processes were included also for nucleonic $S$ - and $P$-wave superfluids. For the $S$-wave $\Lambda$ condensate, we use the rate derived in Paper I. For the $P$-wave hyperonic condensate we adopt the emission rate derived for neutrons (Leinson 2017) with appropriate changes in the condensate parameter and weak charges.

Our strategy in studying the cooling of hypernuclear compact stars is to vary the masses of the stars in the range between 1 to $2 M_{\odot}$. As pointed out in Paper I, there is a mass hierarchy in the cooling behavior, with higher mass stars cooling faster than low mass stars. Another aspect of our study is to vary the input pairing gaps in order to quantify their effect on cooling behavior. We input three nucleonic gaps: the neutron ${ }^{1} S_{0}$, neutron ${ }^{3} P_{2-}{ }^{3} F_{2}$, and proton ${ }^{1} S_{0}$ gap. These form a triplet of nucleonic pairing gaps. Our strategy will be to use alternate gap inputs in such a triplet. The first triplet is defined as SFB-0-CCDK, where the acronyms SFB and CCDK refer to gaps of Schwenk et al. (2003) and Chen et al. (1993), and the number 0 stands for zero pairing gap. The second triplet of pairing gaps is DingS-DingP-CCDK, where DingS and DingP refer to the ${ }^{1} S_{0}$ and ${ }^{3} P_{2}{ }^{3} F_{2}$ gaps obtained by Ding et al. (2016). The hyperonic $\Lambda{ }^{1} S_{0}$ pairing is based on our BCS computation with background matter properties specified by the corresponding EoS. It is added to each triplet of nucleonic gaps. Contrary to what we have done in Paper I, here we disregard pairing of $\Xi$ hyperons. The rationale is to not mask the effect of the high-density pairing of protons and $\Lambda^{\prime}$ 's. $\Sigma^{-}$-pairing is also disregarded. Simulations were conducted with and without $P$-wave pairing for $\Lambda$ 's and protons in the high-density regime in order to quantify their effect; their values were obtained via the scaling procedure described above. In the case of SFB-0-CCDK/DingS-DingP-CCDK, $P$-wave pairing for $\Lambda$ 's and protons is calculated based on neutron $P$-wave pairing of Baldo et al. (1998)/Ding et al. (2016).

Our survey of cooling models shows that they can be separated, broadly speaking, into two groups. The first group which is characterized by low- $L$ values are the Hartree models DDME2 and SWL. The second group associated with large- $L$ values comprises the GM1A model and all the HF models. As shown above, these two groups are characterized by high and low nucleonic dUrca thresholds, respectively. Below we discuss them separately and display for the second group only the results obtained for the PKA1 and PKO3
EoS. Figures 7 and 8 show the cooling tracks for different mass models for these two groups of EoS. For each group, the various panels include or exclude the high-density $P$ wave pairing.

Before turning to the discussion of the features specific to different $\mathrm{EoS}$ it is useful to point out the common features seen in the cooling simulations. (a) There is a mass hierarchy with respect to the speed of cooling - the heavier stars cool faster than the light ones. (b) Neutrino emission due to the PBF processes in a neutron $P$-wave superfluid in the core leads to accelerated cooling after $t \geq 10^{3} \mathrm{yr}$. This acceleration is substantial even for the smallest gaps adopted here (DingP). As a consequence, the cooling tracks undershoot the observational value of the temperature of XMMU J173203.3-344518. Since it is expected that heating, accretion or magnetic fields do not play a role for this object, its high temperature is inconsistent with the existence of a neutron superfluid in the star's core. This motivates the choice of a zero $P$-wave gap in our study. (c) Once dUrca processes are allowed the cooling rate accelerates strongly; the temperatures of the models are then determined by the interplay of several factors: (i) existence of non-superfluid shells within the stars; (ii) effectiveness of suppression of the dUrca process by pairing gaps. The strength of the suppression depends on the magnitude and distribution of the gaps in the star.

Let us now turn to a more detailed discussion of the cooling tracks in Figure 7 for stellar models based on the DDME2 and SWL EoS. Table 3 provides additional information on the reactions which are dominant in a star with a given mass. It also shows the effectiveness of high-density $P$-wave pairing of protons and $\Lambda$ 's in slowing down the cooling. For DDME2 EoS it is seen that, with the exception of the lightest $\left(1 M_{\odot}\right)$ star, all stars cool through the hyperonic dUrca processes and the PBF process in neutron $S$ - and $P$ wave superfluids. (We recall that for the DDME2 EoS the proton fraction is below the nucleonic dUrca threshold so that the dUrca process does not occur in any of these mass models.) Our calculations allow us to extract the density ranges of various processes: $(\Lambda, p)$ channel is active in the density range $0.34 \leq n_{B} \leq 1.02 \mathrm{fm}^{-3}\left(M / M_{\odot} \geq 1.38\right)$; the $\left(\Xi^{-}, \Lambda\right)$ channel is active in the range $0.37 \leq n_{B} \leq 0.98$ $\mathrm{fm}^{-3}\left(M / M_{\odot} \geq 1.54\right)$; the $\left(\Sigma^{-}, \Lambda\right)$ channel is active in the range $0.39 \leq n_{B} \leq 0.60 \mathrm{fm}^{-3}\left(1.60 \leq M / M_{\odot} \leq 2\right)$. Note that here and in all models that will be discussed below the $\left(\Sigma^{-}, n\right)$ channel is energetically forbidden. This is not surprising given the large difference in their abundances.

We observe that the mass range $1 \leq M / M_{\odot} \leq 1.85$ covers the observed range of temperature well, showing a clear mass hierarchy of the cooling behavior of NSs in the neutrino cooling era $t \leq 10^{5} \mathrm{yr}$. [Note that this hierarchy is inverted at the very early $(t \leq 10 \mathrm{yr})$ stages of thermal evolution, which is however observationally insignificant].

Firstly, we note that switching on and off the neutron $P$-wave pairing has an important effect on the cooling of low-to-intermediate mass stars as this pairing significantly reduces the heat capacity of the core and induces one of the dominant cooling processes via PBF. For the late cooling era $t \geq 10^{5} \mathrm{yr}$, we find that the cooling tracks computed for zero neutron $P$-wave pairing gaps are in better agreement with the data than the cooling tracks computed for nonzero gaps. [Note that the youngest stars such as the compact 
central objects (CCO) in Cass A with an estimated age of $t \sim 330$ yr or XMMU J173203.3-344518 with $t \sim 2.7 \times 10^{3}$ yr may have light elements in their atmospheres. Therefore, they may have higher temperatures than predicted by our models.] As can be seen from Table 3, the hyperonic dUrca processes operate in all $M / M_{\odot} \geq 1.38$ stars. Stars with $1.40 \leq M / M_{\odot} \leq 1.6$ cool down via $(\Lambda, p)$, which is the only active dUrca process in their interiors. In contrast to this, several reactions are triggered in stars with masses of $M / M_{\odot} \geq 1.7$, with $\left(\Sigma^{-}, \Lambda\right)$ being the dominant one. Nevertheless, the cooling of stars with $M / M_{\odot} \leq 1.85$ is not fast because of the presence of a proton and/or $\Lambda$ superfluidity.

Increasing the mass of the star increases its central density. For a sufficiently massive star, its central density can exceed the density at which the $\Lambda S$-wave gap disappears. In this case the stars cool fast by the $\left(\Sigma^{-}, \Lambda\right)$ dUrca processes because at high densities $\Lambda$ 's are unpaired. However, if one allows for a higher $P$-wave pairing of $\Lambda$ 's, it slows down the cooling of the $M / M_{\odot}=2$ model, as seen Fig. 7. The $\Lambda P$ wave pairing scaled from neutron $P$-wave pairing results of Ding et al. (2016) (right panel) is less effective than the one obtained from that of Baldo et al. (1998) (left panel). The reason is that the latter spans a wider range of densities and its maximum is located at a larger value (see Fig. 4).

Next, we consider the cooling tracks computed for the SWL EoS. They are shown in the lower part of Fig. 7. This case is similar to DDME2 considered above, as the nucleonic dUrca process does not occur in stars with masses $M / M_{\odot} \leq$ 2 . From our models the density ranges of hyperonic dUrca processes are as follows: $(\Lambda, p)$ channel operates in the density range $0.41 \leq n_{B} \leq 0.94 \mathrm{fm}^{-3}\left(1.5 \leq M / M_{\odot} \leq 2\right)$, $\left(\Xi^{-}, \Lambda\right)$ operates in the range $0.45 \leq n_{B} \leq 0.94 \mathrm{fm}^{-3}$ $\left(1.66 \leq M / M_{\odot} \leq 2\right)$. As was the case for the DDME2 EoS keeping or switching off the $P$-wave pairing of neutrons affects the cooling of low-to-intermediate mass stars due to the presence or absence of the corresponding PBF process. It can be seen that the zero gap case reproduces the measured surface temperatures of mature $t \geq 10^{5} \mathrm{NSs}$. Because hyperonic dUrca processes are dominant in stars with masses $M / M_{\odot} \geq 1.6$ (see Table 3 ) the cooling tracks of stars with masses below this value are practically indistinguishable from each other and produce the highest temperatures. Once the hyperonic dUrca processes set in there is a mild drop in the cooling tracks of stars of masses $M / M_{\odot} \leq 1.8$, produced by the balance between the large neutrino emissivities of these hyperonic dUrca processes and the suppression via hyperon and proton $S$-wave pairing. The onset of the reaction $\left(\Xi^{-}, \Lambda\right)$, which is only partially suppressed, accelerates the cooling of the most massive stars, leading to a significant drop in their temperature. $P$-wave pairing of $\Lambda$ 's results in higher temperatures of stars with $M / M_{\odot} \geq 1.9$, for it diminishes the emissivity of both $\left(\Xi^{-}, \Lambda\right)$ and $(\Lambda, p)$. Contrary to what we have seen for the case DDME2 EoS based models, for the SWL EoS based models the $P$-wave pairing of protons plays a role too when the proton gap is large enough, as it happens when the gap is obtained from scaling the neutron $P$-wave pairing gap of Baldo et al. (1998). This case is not illustrated in Fig. 7 as it is similar to the case of $\Lambda$-P wave pairing. 

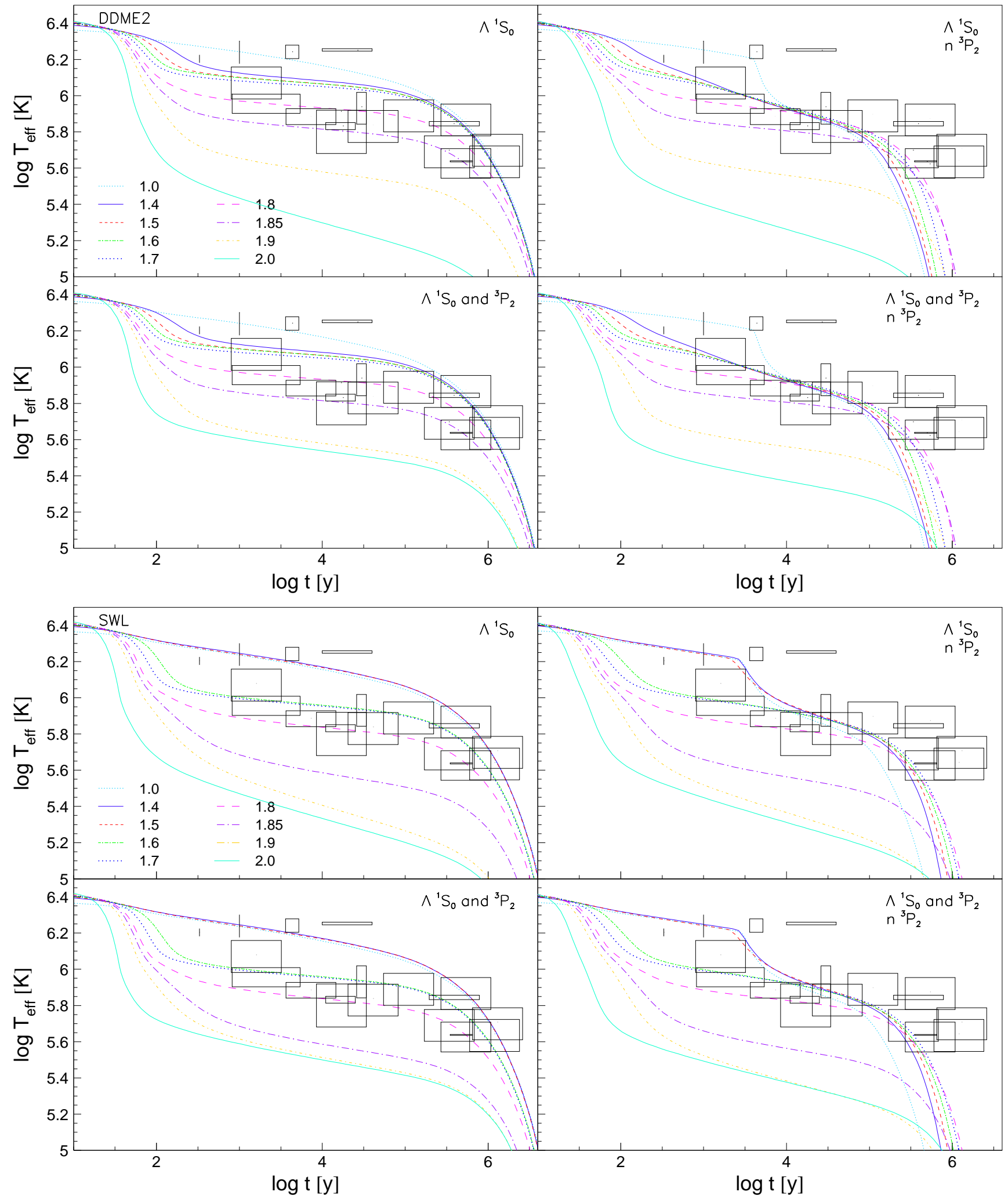

Figure 7. Cooling models based on the DDME2 EoS (upper two rows) and SWL EoS (lower two rows) for NS masses within the range $1 \leq M / M_{\odot} \leq 2$. The left panels use for the triplet of neutron ${ }^{1} S_{0}$ and ${ }^{3} P_{2^{-}}{ }^{3} F_{2}$ and proton ${ }^{1} S_{0}$ gaps of/associated with SFB-0-CCDK, where SFB refers to Schwenk et al. (2003) and CCDK to Chen et al. (1993), and 0 indicates that the neutron $P$-wave pairing gap is zero. The right panels use the DingS-DingP-CCDK triplet. The $\Lambda{ }^{1} S_{0}$ pairing gaps are based on our BCS computations. The $\Lambda$ and proton $P$-wave gaps are obtained from the scaling procedure described in the text using for the neutron $P$-wave the results of Baldo et al. (1998) (left column) and, respectively, Ding et al. (2016) (right column). 
Table 3. List of reactions which dominate the cooling evolution (columns 2 and 5) and efficiency of high density pairing of protons and $\Lambda$ s (indicated by - (zero efficiency) or $\sqrt{ }$ (non-zero efficiency) in columns $3,4,6,7)$ in NS models of various masses built upon EoS which do not allow for nucleonic dUrca, DDME2 (upper part) and SWL (lower part), for any of the considered masses. Columns 2-4 (5-7) correspond to the set SFB-0-CCDK (DingS-DingP-CCDK) for neutron ${ }^{1} S_{0}$ and ${ }^{3} P_{2^{-}}{ }^{3} F_{2}$ and proton ${ }^{1} S_{0}$ gaps, see text. The $\Lambda$ ${ }^{1} S_{0}$ gaps are based on our BCS computations assuming a background given by the corresponding EoS. The proton and $\Lambda{ }^{3} P_{2}$ gaps are obtained by scaling the neutron $P$-wave gap by Baldo et al. (1998) (columns 3-4) or, alternatively, by Ding et al. (2016) (columns 6-7). The abbreviations are as follows: PBF stands for Cooper pair breaking-formation processes; $n\left({ }^{3} P_{2}\right)$ neutron $P$-wave pairing and similarly for other baryons and channels. Baryons in brackets, such as $(\Lambda, p)$ stand for direct Urca process involving indicated baryons [see Eqs. (3)-(6)]. N bremstr. stands for nucleonic bremsstrahlung.

\begin{tabular}{|c|c|c|c|c|c|c|}
\hline$M / M_{\odot}$ & dominant reactions & $p\left({ }^{3} P_{2}\right)$ & $\Lambda\left({ }^{3} P_{2}\right)$ & dominant reactions & $p\left({ }^{3} P_{2}\right)$ & $\Lambda\left({ }^{3} P_{2}\right.$ \\
\hline & \multicolumn{3}{|c|}{ no $n\left({ }^{3} P_{2}\right)$} & \multicolumn{3}{|l|}{$n\left({ }^{3} P_{2}\right)$} \\
\hline \multicolumn{7}{|l|}{ DDME2 } \\
\hline 1.0 & PBF_n ${ }^{1} S_{0}, \mathrm{~N}$ bremstr. & - & - & PBF_n ${ }^{3} P_{2}, \mathrm{~N}$ bremstr. & - & - \\
\hline 1.4 & $(\Lambda, p)$, PBF_n ${ }^{1} S_{0}$ & - & - & $(\Lambda, p)$, PBF $\_\mathrm{n}^{3} P_{2}$ & - & - \\
\hline 1.5 & $(\Lambda, p)$, PBF_n ${ }^{1} S_{0}$ & - & - & $(\Lambda, p)$, PBF_n ${ }^{3} P_{2}$ & - & - \\
\hline 1.6 & $(\Lambda, p)$, PBF_n ${ }^{1} S_{0}$ & - & - & $(\Lambda, p)$, PBF_n ${ }^{3} P_{2}$ & - & - \\
\hline 1.7 & $(\Lambda, p),\left(\Sigma^{-}, \Lambda\right)$, PBF_n ${ }^{1} S_{0}$ & - & - & $(\Lambda, p)$, PBF_n ${ }^{3} P_{2}$ & - & - \\
\hline 1.8 & $\left(\Sigma^{-}, \Lambda\right)$, PBF_n ${ }^{1} S_{0}$ & - & - & $\left(\Sigma^{-}, \Lambda\right),(\Lambda, p)$, PBF_n ${ }^{3} P_{2}$ & - & - \\
\hline 1.9 & $\left(\Sigma^{-}, \Lambda\right)$ & - & - & $\left(\Sigma^{-}, \Lambda\right),(\Lambda, p)$ & - & - \\
\hline 2.0 & $\left(\Sigma^{-}, \Lambda\right)$ & - & $\sqrt{ }$ & $\left(\Sigma^{-}, \Lambda\right),(\Lambda, p)$ & - & $\sqrt{ }$ \\
\hline \multicolumn{7}{|l|}{ SWL } \\
\hline 1.3 & PBF_n $\mathrm{n}^{1} S_{0}, \mathrm{~N}$ bremstr. & - & - & PBF_n ${ }^{3} P_{2}, \mathrm{~N}$ bremstr. & - & - \\
\hline 1.4 & PBF_n ${ }^{1} S_{0}, \mathrm{~N}$ bremstr. & - & - & PBF_n ${ }^{3} P_{2}, \mathrm{~N}$ bremstr. & - & - \\
\hline 1.5 & PBF_n ${ }^{1} S_{0}, \mathrm{~N}$ bremstr. & - & - & PBF_n ${ }^{3} P_{2}, \mathrm{~N}$ bremstr. & - & - \\
\hline 1.6 & $(\Lambda, p)$, PBF $\_\mathrm{n}^{1} S_{0}$ & - & - & $(\Lambda, p)$, PBF $\_\mathrm{n}^{3} P_{2}$ & - & - \\
\hline 1.7 & $(\Lambda, p)$, PBF_n ${ }^{1} S_{0}$ & - & - & $(\Lambda, p)$, PBF_n ${ }^{3} P_{2}$ & - & - \\
\hline 1.8 & $(\Lambda, p),\left(\Xi^{-}, \Lambda\right)$ & - & - & $(\Lambda, p),\left(\Xi^{-}, \Lambda\right)$, PBF $\_n^{3} P_{2}$ & - & - \\
\hline 1.9 & $(\Lambda, p),\left(\Xi^{-}, \Lambda\right)$ & $\sqrt{ }$ & $\sqrt{ }$ & $(\Lambda, p),\left(\Xi^{-}, \Lambda\right)$ & - & $\sqrt{ }$ \\
\hline 2.0 & $(\Lambda, p),\left(\Xi^{-}, \Lambda\right)$ & $\sqrt{ }$ & $\sqrt{ }$ & $(\Lambda, p),\left(\Xi^{-}, \Lambda\right)$ & - & $\sqrt{ }$ \\
\hline
\end{tabular}



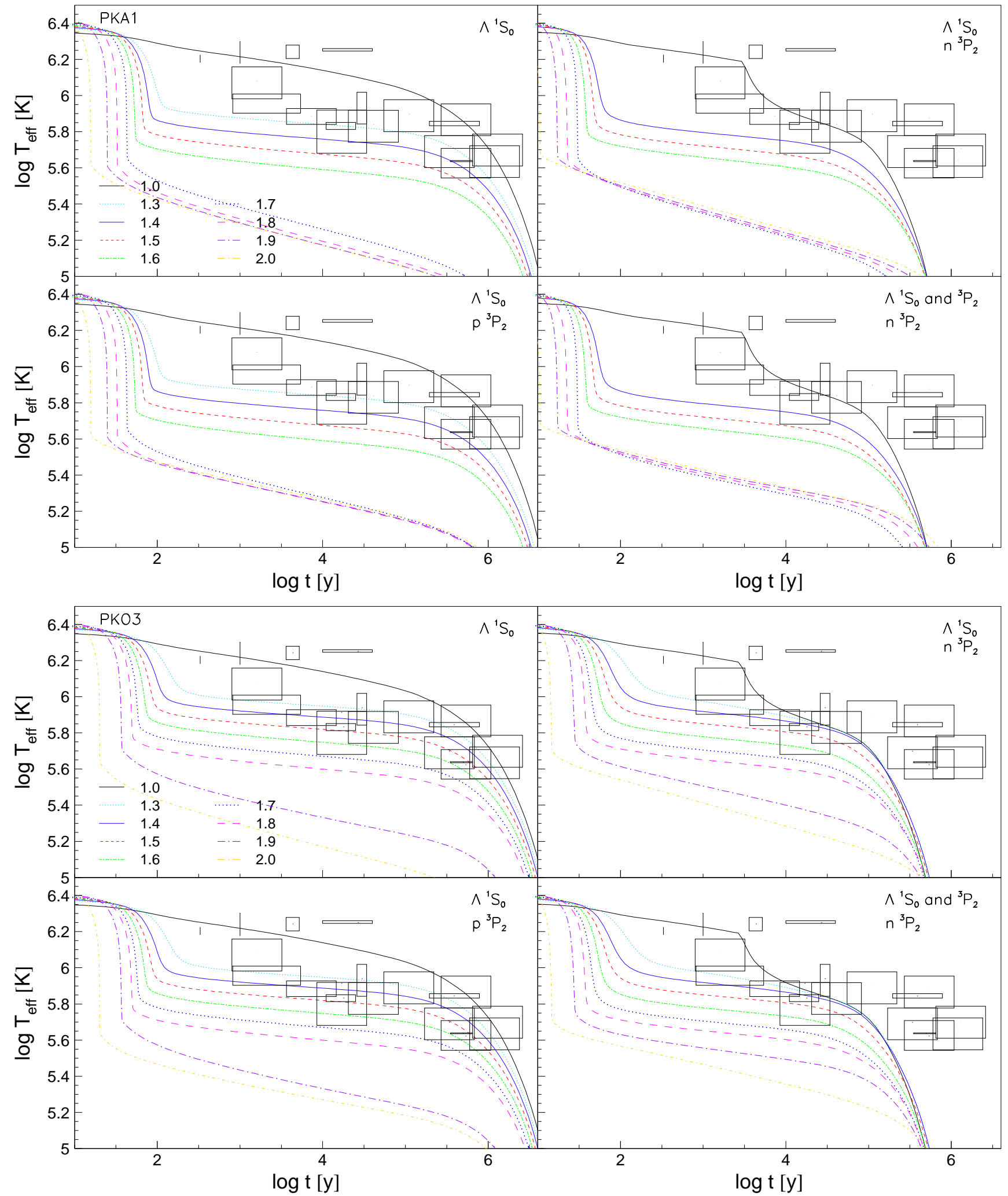

Figure 8. Cooling models based on the PKA1 EoS (upper two rows) and PKO3 EoS (lower two rows) for NS masses within the range $1 \leq M / M_{\odot} \leq 2$. The left panels use for the triplet of neutron ${ }^{1} S_{0}$ and ${ }^{3} P_{2^{-}}{ }^{3} F_{2}$ and proton ${ }^{1} S_{0}$ gaps of/associated with SFB-0-CCDK, where the acronyms SFB refers to Schwenk et al. (2003) and CCDK to Chen et al. (1993), and 0 stands for zero pairing gap. The right panels use the following triplet of pairing gaps: DingS-DingP-CCDK, where DingS and DingP refer to the ${ }^{1} S_{0}$ and ${ }^{3} P_{2-}{ }^{3} F_{2}$ gaps obtained by Ding et al. (2016). The $\Lambda^{1} S_{0}$ pairing gap are based on our BCS computations. The $\Lambda$ and proton $P$-wave gaps are obtained from the scaling procedure described in the text using for the neutron $P$-wave the results of Baldo et al. (1998) (left column) and, respectively, Ding et al. (2016) (right column) 
The cooling tracks for the PKA1 and PKO3 EoS are shown in Fig. 8. Compared to the DDME2 and SWL based models discussed above, the new qualitative feature is the low threshold for the nucleonic direct Urca process.

We extract from our PKA1 EoS a density threshold of $n_{B} \geq 0.25 \mathrm{fm}^{-3}$ above which the $(n, p)$ reaction is effective. This density corresponds to $M / M_{\odot} \geq 0.98$, which means that all models considered allow for nucleonic dUrca. Additional contributions come from the hyperonic dUrca processes, where $(\Lambda, p)$ is effective over the density range $0.32 \leq$ $n_{B} \leq 0.95 \mathrm{fm}^{-3}\left(1.44 \leq M / M_{\odot} \leq 1.99\right),\left(\Sigma^{-}, \Lambda\right)$ is effective over the range $0.40 \leq n_{B} \leq 0.47 \mathrm{fm}^{-3}\left(1.59 \leq M / M_{\odot} \leq\right.$ $1.67)$, and $\left(\Xi^{-}, \Lambda\right)$ acts over the range $0.45 \leq n_{B} \leq 0.95$ $\mathrm{fm}^{-3}\left(1.64 \leq M / M_{\odot} \leq 1.99\right)$. The cooling tracks, illustrated in the top panels of Fig. 8, fall into three classes. The first class is represented by the lightest star. With a core that accommodates nucleonic dUrca over a volume of insignificant size, the $M / M_{\odot}=1$ model manifests slow cooling, via neutrino emission from nucleon bremsstrahlung and PBF from neutron pairing in $S$ - and $P$-channels. The second class of models, with $1.3 \leq M / M_{\odot} \leq 1.6$, cool down exclusively or predominantly by the nucleonic dUrca process. As at least one of the involved species, i.e. protons, $\Lambda \mathrm{s}$ or - as in the case for the panels on the right - neutrons, is paired, the cooling is moderate and the cooling tracks cover a domain in the $t-T_{\text {eff }}$ plane where observed data exist or are not far from them. The third class is represented by models with $M / M_{\odot} \geq 1.7$. Their fast cooling is due to unpaired baryons, protons (left panels of Fig. 8) and $\Lambda \mathrm{s}$ (right panels of Fig. 8). As already discussed in relation with the DDME2- and SWL-models, even a small neutron $P$-wave pairing leads to accelerated cooling for $t \gtrsim 10^{3} \mathrm{yr}$, irrespective of the star's mass. According to Table 4, even a tiny neutron $P$-wave pairing gap (or, equivalently $T_{c}$ ) as the one of Ding et al. (2016) is sufficient to switch the dominant dUrca from nucleonic (left panels, no neutron $P$-pairing) to hyperonic (right panels, with neutron $P$-pairing).

Switching on and off the $P$-wave superfluidity in the proton and $\Lambda$ components does not change the general behavior of cooling tracks qualitatively. It is seen that most massive stars with $P$-wave superfluidity have higher temperatures compared to their counterparts without such pairing. Depending on whether or not neutron $P$-wave pairing occurs, the cooling of these stars is slowed down by proton- or, alternatively, $\Lambda$ - $P$-wave pairing.

The models based on the PKO3 EoS, shown in the bottom panels of Fig. 8, are qualitatively similar to those based on the PKA1 EoS. The reason for this lies in the relatively low nucleonic dUrca threshold, $n_{B} \geq 0.28 \mathrm{fm}^{-3}$, which makes this channel effective in models with $M / M_{\odot} \geq 1.22$. The $(\Lambda, p)$ and $\left(\Xi^{-}, \Lambda\right)$ hyperonic dUrca processes act over baryonic density ranges of $0.33 \leq n_{B} \leq 0.86 \mathrm{fm}^{-3}$ and $0.54 \leq n_{B} \leq 0.83 \mathrm{fm}^{-3}$, respectively, which correspond to NS masses domains of $1.56 \leq M / M_{\odot} \leq 1.99$ and $1.89 \leq M / M_{\odot} \leq 1.99$.

As for PKA1 EoS based models, the nucleonic dUrca in stars with $M / M_{\odot} \geq 1.8$ is suppressed by the neutron $P$-wave pairing; as a consequence, the hyperonic dUrca, in this case $(\Lambda, p)$, is as efficient as the nucleonic dUrca. As illustrated in Fig. 2, PKO3 EoS leads to lower proton- and $\Lambda$-abundances than PKA1 EoS. As a consequence, the ${ }^{1} S_{0^{-}}$ pairing of protons and $\Lambda$ 's computed for stellar models based on the PKO3 EoS extends over larger core regions than is the case for models based on the PKA1 EoS. They are additionally characterized by higher critical temperatures, as can be seen in Figs. 5 and 6 . These facts explain the quantitative differences between the cooling curves associated with the two EoS. The separation between slow-, intermediateand fast-cooling tracks is less pronounced for PKO3 than for PKA1 based EoS. We also note that for a given stellar mass and fixed set of pairing-gaps, the effective temperatures are higher. The lower densities of protons and $\Lambda$ 's produced by PKO3 render the high-density pairing of these particles less efficient. Indeed, the drop in the temperature becomes smaller only for models with $M / M_{\odot}=1.9$ and 2 when $\Lambda$ $P$-wave pairing is additionally taken into account; this occurs only for $M / M_{\odot}=2$ models if proton $P$-wave pairing is included in addition.

\section{CONCLUSIONS}

This work extends the previous study of Raduta et al. (2018) to the cooling of hypernuclear stars by including models for the EoS based on the Hartree-Fock treatment of the hypernuclear matter. We conjecture on the existence of new channels of $P$-wave pairing in the proton and $\Lambda$ components of our models and explore their effect on the cooling behavior of NSs using simple scaling relations for the pairing gaps. The EoS models can be classified into two groups: the first group, which includes the DDME2 and SWL models, has a low- $L$ value and does not allow for nucleonic dUrca or allows for it only in very massive stars. The second group consists of GM1A and all HF models, which have a high $L$ value but a rather low nucleonic dUrca threshold.

The grouping of the models in two classes reflects the uncertainties in predictions of the current density functionals of the $L$-value, which is not well constrained. The variations in the $L$ value predicted by different models can be traced back to the fit parameters that are used to construct any particular density functional, in particular, the relative weight of observables that belong to iso-vector and iso-scalar sectors. In low- $L$ density functionals (e.g. the DDME2 functional) the iso-vector probes have a significant weight in the data to which the parameters are tuned. On the contrary, the family of Hartree-Fock PK-models, which predict large $L$-values, has been designed using fits to experimental data of binding energies of magic nuclei, spin-orbit splittings of neutron and proton $1 \mathrm{p}$ states of ${ }^{16} \mathrm{O}$, shell gaps at $Z=58$ and 64 and properties of symmetric saturated matter.

- Mass hierarchies and observational data. We find in general that all the models follow a mass-hierarchy. If one arranges the stars from the lightest to the heaviest ones, the former cool always slower than the latter in the neutrino cooling era $t<10^{5}$ yr. Mass hierarchies have been observed for a long time, in particular in the studies where some fast cooling agent has been included; for example, this is the case when stars contain a quark core (Page et al. 2000; Grigorian et al. 2005; Blaschke et al. 2012; Hess \& Sedrakian 2011; Sedrakian 2013) or cool by direct Urca process (Schaab et al. 1997; Taranto et al. 2016). Such a hierarchy has also been also obtained in the studies based on the medium-modified cooling scenario; for a recent discussion see (Grigorian et al. 2018). The mass hierarchy 
Table 4. Same as in Table 3 but for the PKA1 and PKO3 EoS.

\begin{tabular}{|c|c|c|c|c|c|c|}
\hline$M / M_{\odot}$ & dominant reactions & $p\left({ }^{3} P_{2}\right)$ & $\Lambda\left({ }^{3} P_{2}\right)$ & dominant reactions & $p\left({ }^{3} P_{2}\right)$ & $\Lambda\left({ }^{3} P_{2}\right)$ \\
\hline & \multicolumn{3}{|c|}{ no $n\left({ }^{3} P_{2}\right)$} & \multicolumn{3}{|l|}{$n\left({ }^{3} P_{2}\right)$} \\
\hline \multicolumn{7}{|l|}{ PKA1 } \\
\hline 1.0 & PBF_n ${ }^{1} S_{0}, \mathrm{~N}$ bremstr. & - & - & PBF_n ${ }^{1} S_{0}$, PBF_n ${ }^{3} P_{2}, N$ bremstr. & - & - \\
\hline 1.4 & $(n, p)$ & - & - & $(n, p)$ & - & - \\
\hline 1.5 & $(n, p)$ & - & - & $(n, p)$ & - & - \\
\hline 1.6 & $(n, p)$ & - & - & $(n, p),(\Lambda, p)$ & - & - \\
\hline 1.7 & $(n, p),\left(\Sigma^{-}, \Lambda\right)$ & $\sqrt{ }$ & $\sqrt{ }$ & $\left(\Sigma^{-}, \Lambda\right)$ & - & $\sqrt{ }$ \\
\hline 1.8 & $(n, p)$ & $\sqrt{ }$ & - & $\left(\Sigma^{-}, \Lambda\right)$ & - & $\sqrt{ }$ \\
\hline 1.9 & $(n, p)$ & $\sqrt{ }$ & - & $\left(\Sigma^{-}, \Lambda\right)$ & - & $\sqrt{ }$ \\
\hline 2.0 & $(n, p)$ & $\sqrt{ }$ & - & $(\Lambda, p),\left(\Sigma^{-}, \Lambda\right),\left(\Xi^{-}, \Lambda\right)$ & - & $\sqrt{ }$ \\
\hline \multicolumn{7}{|l|}{ PKO3 } \\
\hline 1.0 & PBF_n ${ }^{1} S_{0}, \mathrm{~N}$ bremstr. & - & - & PBF_n ${ }^{3} P_{2}, \mathrm{~N}$ bremstr. & - & - \\
\hline 1.4 & $(n, p)$ & - & - & $(n, p)$, PBF_n $\mathrm{n}^{3} P_{2}$ & - & - \\
\hline 1.5 & $(n, p)$ & - & - & $(n, p)$, PBF_n ${ }^{3} P_{2}$ & - & - \\
\hline 1.6 & $(n, p)$ & - & - & $(n, p)$ & - & - \\
\hline 1.7 & $(n, p)$ & - & - & $(n, p)$ & - & - \\
\hline 1.8 & $(n, p)$ & - & - & $(n, p),(\Lambda, p)$ & - & - \\
\hline 1.9 & $(n, p)$ & - & - & $(n, p),(\Lambda, p)$ & - & $\sqrt{ }$ \\
\hline 2.0 & $(n, p)$ & $\sqrt{ }$ & - & $(\Lambda, p),\left(\Xi^{-}, \Lambda\right)$ & - & $\sqrt{ }$ \\
\hline
\end{tabular}

may, however, be inverted at a very early stage of evolution, i.e., the heavier stars may cool slower than the lighter stars. Similar inversion also is observed at very late stages, when the cooling is dominated by photon emission from the stellar surface (photon cooling era). For the DDME2 and SWL EoS based models, we find that cooling tracks smoothly cover the observationally relevant area in the $t$ - $T_{\text {eff }}$ diagram. Interestingly, we find that vanishing $P$-wave neutron superfluidity improves the agreement with the observational data for our hypernuclear models, a feature already observed in alternative models of cooling evolution of NS. The Hartree-Fock models develop nucleonic dUrca processes rather early. This results in a very fast cooling of these models which renders the agreement with the data difficult. Nevertheless, for the PKO3 model, we find that light to medium-mass stars have cooling tracks that pass through the observationally relevant region in the $t$ - $T_{\text {eff }}$ diagram.

- High-density pairing. In this part of the work, we attempted to rectify the too rapid cooling of the most massive NS models of our study, which is linked to the closing of the ${ }^{1} S_{0}$ gaps for $\Lambda$ 's and protons at high densities. We have proposed simple scaling arguments for obtaining the gaps of these particles from those computed for neutron matter. From this, we found that if $P$-wave pairing of protons and $\Lambda$ 's is allowed, then even the most massive $\left(1.9 \leq M / M_{\odot} \leq 2\right)$ hypernuclear stars will undergo a significantly slower cooling evolution.

The diversity among presently considered hypernuclear EoS, which leads to differences in the thermal evolution of NSs, is partly rooted in the uncertainties in the nucleonic sector ( $L$-value and, very probably, higher order terms of the expansion of the symmetry energy as a function of scalar density), the assumed spin-flavor symmetry of the quark model which controls the coupling of hyperons to nucleons in the vector meson sector, and the uncertainty in the val- ues of the hyperon-nucleon interaction potential which does the same in the scalar meson sector. An interesting possibility not studied so far is a lower value of $\Sigma$ potential in the nuclear matter than assumed here. In that case, already relatively light mass stars will experience fast cooling via $\Sigma^{-} \rightarrow \Lambda+l+\bar{\nu}_{l}$, even if $\Lambda$-pairing is taken into account. For DDME2 our preliminary results show that models with $M / M_{\odot} \geq 1.5$ manifest strong disagreement with the data. The situation might nevertheless significantly improve if one additionally allows for $\Sigma$-pairing. These scenarios are under consideration and will be published elsewhere. Despite of this diversity, our study elucidates how various ingredients of microphysical input (parametrization of the EoS, pairing gaps, etc.) affect the cooling evolution of neutron stars. This establishes controlled correspondence between various input parameters and the general behavior of cooling tracks of hypernuclear stars, which can be used as a diagnostics of the internal composition of NSs in the future. While a general picture of the cooling of hypernuclear star is emerging, the systematic coverage of the parameter space remains a task for the future. This would include the empirical parameters of nuclear matter at saturation, the range of pairing gaps predicted by the many-body theory, as well as an estimate of the effect of the statistical uncertainties that are inherent to the microphysics input on the cooling simulations.

\section{ACKNOWLEDGMENTS}

A. R. acknowledges the support provided by the European COST Actions "NewCompStar" (MP1304) and "PHAROS" (CA16214), through STSM grants as well as the hospitality of the Frankfurt Institute for Advanced Studies. J.-J. Li was supported by Alexander von Humboldt Foundation. A. S. is supported by the Deutsche Forschungsgemeinschaft (Grant No. SE 1836/4-1). F. W. is supported by the National Sci- 
ence Foundation (USA) under Grant PHY-1714068. This research has made use of NASA's Astrophysics Data System.

\section{REFERENCES}

Abbott B. P., et al. 2017, Phys. Rev. Lett., 119, 161101

Akmal A., Pandharipande V. R., Ravenhall D. G., 1998, Phys. Rev. C, 58, 1804

Annala E., Gorda T., Kurkela A., Vuorinen A., 2018, Phys. Rev. Lett., 120, 172703

Antoniadis J., et al., 2013, Science, 340, 448

Balberg S., Barnea N., 1998, Phys. Rev. C , 57, 409

Baldo M., Elgaroy O., Engvik L., Hjorth-Jensen M., Schulze H.J., 1998, Phys. Rev. C, 58, 1921

Barr E. D., Freire P. C. C., Kramer M., Champion D. J., Berezina M., Bassa C. G., Lyne A. G., Stappers B. W., 2017, MNRAS, 465,1711

Bednarek I., Haensel P., Zdunik J., Bejger M., Mańka R., 2012, Astron. Astrophys., 543, A157

Beznogov M. V., Rrapaj E., Page D., Reddy S., 2018, Phys. Rev. C, 98, 035802

Blaschke D., Grigorian H., Voskresensky D. N., 2004, Astron. Astrophys., 424, 979

Blaschke D., Grigorian H., Voskresensky D. N., Weber F., 2012, Phys. Rev. C , 85, 022802

Bonanno L., Sedrakian A., 2012, Astron. Astrophys., 539, A16

Chao N.-C., Clark J., Yang C.-H., 1972, Nuclear Physics A, 179, 320

Chen J., Clark J., Dave R., Khodel V., 1993, Nucl. Phys. A, 555, 59

Christian J.-E., Zacchi A., Schaffner-Bielich J., 2019, Phys. Rev. D , 99, 023009

Colucci G., Sedrakian A., 2013, Phys. Rev. C, 87, 055806

Demorest P. B., Pennucci T., Ransom S. M., Roberts M. S. E., Hessels J. W. T., 2010, Nature, 467, 1081

Ding D., Rios A., Dussan H., Dickhoff W. H., Witte S. J., Carbone A., Polls A., 2016, Phys. Rev. C, 94, 025802

Fattoyev F. J., Piekarewicz J., Horowitz C. J., 2018, Phys. Rev. Lett., 120, 172702

Filikhin I., Gal A., 2002, Nucl. Phys. A, 707, 491

Fonseca E., Pennucci T. T., Ellis J. A., Stairs I. H., Nice D. J., et al., 2016, ApJ, 832, 167

Fortin M., Providência C., Raduta A. R., Gulminelli F., Zdunik J. L., Haensel P., Bejger M., 2016, Phys. Rev. C, 94, 035804

Fortin M., Avancini S. S., Providência C., Vidaña I., 2017, Phys. Rev. C, 95, 065803

Gal A., Hungerford E. V., Millener D. J., 2016, Rev. Mod. Phys., 88, 035004

Gomes R. O., Dexheimer V., Schramm S., Vasconcellos C. A. Z., 2015, ApJ, 808, 8

Grigorian H., Blaschke D., Voskresensky D., 2005, Phys. Rev. C , 71,045801

Grigorian H., Voskresensky D., Maslov K., 2018, Nucl. Phys. A, 980, 105

Gusakov M. E., Haensel P., Kantor E. M., 2014, MNRAS, 439, 318

Haensel P., Gnedin O. Y., 1994, Astron. Astrophys., 290, 458

Haensel P., Zdunik J. L., Dobaczewski J., 1989, Astron. Astrophys., 222, 353

Hess D., Sedrakian A., 2011, Phys. Rev. D , 84, 063015

Kucharek H., Ring P., 1991, Z. Phys. A, 339, 23

Lattimer J. M., Steiner A. W., 2014, Eur. Phys. J. A, 50, 40

Leinson L. B., 2017, arXiv e-prints: 1712.10214 ,

Li J. J., Long W. H., Sedrakian A., 2018a, Eur. Phys. J. A, 54, 133

Li J. J., Sedrakian A., Weber F., 2018b, Phys. Lett. B, 783, 234
Lonardoni D., Lovato A., Gandolfi S., Pederiva F., 2015, Phys. Rev. Lett., 114, 092301

Long W. H., Sun B. Y., Hagino K., Sagawa H., 2012, Phys. Rev. C, 85, 025806

Machleidt R., Sammarruca F., Song Y., 1996, Phys. Rev. C, 53, R1483

Marques M., Oertel M., Hempel M., Novak J., 2017, Phys. Rev. C, 96, 045806

Maslov K., Kolomeitsev E., Voskresensky D., 2015, Phys. Lett. B, 748, 369

Miyatsu T., Cheoun M.-K., Saito K., 2013, Phys. Rev. C, 88, 015802

Most E. R., Weih L. R., Rezzolla L., Schaffner-Bielich J., 2018, Phys. Rev. Lett., 120, 261103

Negele J., Vautherin D., 1973, Nucl. Phys. A, 207, 298

Negreiros R., Tolos L., Centelles M., Ramos A., Dexheimer V., 2018, ApJ, 863, 104

Oertel M., Providência C., Gulminelli F., Raduta A. R., 2015, J. Phys. G, 42, 075202

Page D., Prakash M., Lattimer J. M., Steiner A. W., 2000, Physical Review Letters, 85, 2048

Page D., Lattimer J. M., Prakash M., Steiner A. W., 2004, ApJ Supplement Series, 155, 623

Paschalidis V., Yagi K., Alvarez-Castillo D., Blaschke D. B., Sedrakian A., 2018, Phys. Rev. D , 97, 084038

Prakash M., Prakash M., Lattimer J. M., Pethick C. J., 1992, ApJ Lett., 390, L77

Providencia C., Fortin M., Pais H., Rabhi A., 2018

Raduta A. R., Sedrakian A., Weber F., 2018, MNRAS, 475, 4347

Rijken T. A., 2001, Nucl. Phys. A, 691, 322

Schaab C., Voskresensky D., Sedrakian A. D., Weber F., Weigel M. K., 1997, Astron. Astrophys., 321, 591

Schaab C., Balberg S., Schaffner-Bielich J., 1998, ApJ Letters, 504, L99

Schwenk A., Friman B., Brown G. E., 2003, Nucl. Phys. A, 713, 191

Sedrakian A., 2013, A\&A, 555, L10

Sedrakian A., Clark J. W., 2018, arXiv e-prints: 1802.00017

Spinella W., 2017, PhD thesis, Claremont Graduate University/San Diego State University

Spinella W. M., Weber F., 2018, arXiv e-prints: 1812.03600,

Stone J. R., Guichon P., Matevosyan H., Thomas A., 2007, Nuclear Physics A, 792, 341

Takatsuka T., Nishizaki S., Yamamoto Y., Tamagaki R., 2001, Prog. Theor. Phys., 105, 179

Taranto G., Burgio G. F., Schulze H.-J., 2016, MNRAS, 456, 1451

Tews I., Margueron J., Reddy S., 2019, arXiv e-prints: 1901.09874

Togashi H., Hiyama E., Yamamoto Y., Takano M., 2016, Phys. Rev. C, 93, 035808

Tolos L., Centelles M., Ramos A., 2016, ApJ, 834, 3

Tolos L., Centelles M., Ramos A., 2017a, Publ. Astron. Soc. Austral., 34, e065

Tolos L., Centelles M., Ramos A., 2017b, ApJ, 834, 3

Tsang M. B., et al., 2012, Phys. Rev. C, 86, 015803

Tsuruta S., Sadino J., Kobelski A., Teter M. A., Liebmann A. C., Takatsuka T., K.Nomoto Umeda H., 2009, ApJ, 691, 621

Van Dalen E., Colucci G., Sedrakian A., 2014, Phys. Lett. B, 734, 383

Vidaña I., Tolós L., 2004, Phys. Rev. C , 70, 028802

Vretenar D., Afanasjev A. V., Lalazissis G. A., Ring P., 2005, Phys. Rep., 409, 101

Wang Y. N., Shen H., 2010, Phys. Rev. C, 81, 025801

Weissenborn S., Chatterjee D., Schaffner-Bielich J., 2012a, Phys. Rev. C, 85, 065802

Weissenborn S., Chatterjee D., Schaffner-Bielich J., 2012b, Nucl. Phys. A, 881, 62

Wiringa R. B., Stoks V. G. J., Schiavilla R., 1995, Phys. Rev. C, 51,38 
Yamamoto Y., Togashi H., Tamagawa T., Furumoto T., Yasutake N., Rijken T. A., 2017, Phys. Rev. C, 96, 065804 